\title{
TOM EFFECTS DURING QUIET AND TURBULENT TIMES
}

\author{
Ramona Dumitriu \\ rdumitriu@ugal.ro \\ Razvan Stefanescu \\ rzvn_stefanescu@yahoo.com \\ University "Dunarea de Jos" of Galati \\ Faculty of Economics and Business Administration
}

\begin{abstract}
This paper investigates the turn-of-the-month effects presence on stock markets from 32 countries during two periods of time: a relative quiet one from January 2000 to December 2006 and a turbulent one from January 2000 to October 2013. We found some significant changes that occurred from the quiet to the turbulent times and some differences between emerging and developed markets.
\end{abstract}

Keywords: Calendar Anomalies, GARCH, Emerging and Developed Capital Market, Volatility, Persistence in Time

JEL Classification: G02, G14, G19

\section{Introduction}

TOM effect is one of the most known stock markets calendar anomalies consisting in significant differences between the stock returns from the so called TOM period (which includes the first trading days of a month plus the last trading days from the precedent month) and the stock returns from the other days (Ariel, 1987; Thaler, 1987; Lakonishok and Smidt, 1988; Pettengill and Jordan, 1988; Ogden, 1990; Ziemba, 1991; Cadsby, 1992; Martikainen et al., 1994; Hensel et al., 1996; Compton, 2000; Booth, 2001; Kunkel et al., 2003; Joshi and Fatta, 2005; McGuinness, 2006; Xu and McConnell, 2006; Wiley and Zumpano, 2009; Desai and Trivedi, 2012; Liu, 2013). There are many hypotheses that explain this seasonality. Pay Day Hypothesis is based on the fact that usually before TOM period many investors need cash money for some payments such as the dividends, the interests or even the wages of their employees. They obtain money by selling assets which are bought back during TOM period (Ogden, 1987; Ogden, 1990). Window Dressing Hypothesis considers that TOM effect is linked by the tendency of some investment companies to keep in their portfolios, at the end of a month, where usually their activity is analyzed, only assets with high returns, in order to show favorable results. As the new month starts they will buy back the stocks they sold (Haugen and Lakonishok, 1988; Ritter and Chopra, 1989; Lakonishok et al., 1991). Earning Announcement Hypothesis explains TOM effect by the fact that most of the announcements about the firms' financial results, which have significant effects on the stock prices, are released during the last days of the month (Penman, 1987; Ball and Kothari, 1991). United States Macroeconomic News Announcement Hypothesis is based on the fact that major announcements regarding US economy, which could influence most of the international financial markets, occur around TOM period (Graham et al., 2003; Nikkinen and Sahlstrom, 2004; Nikkinen et al., 2007).

Like other calendar anomalies, TOM effect could be exploited by investment strategies on the stock markets that are not consistent with Fama (1970) Efficient Market Hypothesis. However such strategies are successful only if this seasonality is persistent in time. Empirical researches found that many calendar anomalies are changing in time (Dimson and Marsh, 1999; Szakmary and Kiefer, 2004; Marquering et al., 2006). Sometimes, the passing from quiet to turbulent periods could affect the stock prices seasonality (Holden et al., 2005). There were also found some differences between seasonality from the developed markets and those from the emerging markets (Wong, 1995; Phylaktis and Ravazzolo, 2002; Li et al., 2003).

The seasonality of stock prices refers not only to their returns but also to their volatility which reflects the risk. The General AutoRegressive Conditional Heteroskedasticity (GARCH) models are among the main tools used in the analysis of the financial markets time-varying volatility (Engle, 1982; Bollersev, 1986). 
In this paper we investigate the presence of TOM effects on 32 stock markets during two different periods of time. The first one, from January 2000 to December 2006, could be considered as a quiet one, while the second, from January 2007 to October 2013 was marked by some processes (the enlargement of European Union, real estate speculative bubble, the global crisis etc.) which induced some significant turbulences on the financial markets. In our investigation we employ GARCH models to reveal the seasonality not only for the indexes returns but also for their volatility. We use the standard GARCH model and two other variants which allow us to capture the asymmetrical reactions of stocks volatility to good and bad news: Glosten et al. (1993) GJR GARCH and Nelson (1991) EGARCH. We analyze the differences between the two periods of time and also between developed and emerging markets.

The rest of this paper is organized as it follows: the second part describes the data and the methodology employed to investigate TOM Effects, the third part presents the empirical results and the fourth part concludes.

\section{Data and Methodology}

In this investigation about the presence of TOM effects we employ daily closing values of the indexes from 32 stock markets (Table 1). Half of them are from the developed countries stock exchanges, while the other half from the emerging markets. In order to reveal the differences between quiet and turbulent times we used two sub-samples of data:

- first sub-sample, corresponding to a relative quiet period, from January 2000 to December 2006;

- second sub-sample, corresponding to a turbulent period, from January 2007 to October 2013.

We calculate, for each index, continuous return $\left(\mathrm{r}_{\mathrm{i}, \mathrm{t}}\right)$ using the formula:

$$
r_{i, t}=\left[\ln \left(P_{i, t}\right)-\ln \left(P_{i, t-1}\right)\right] \times 100
$$

where $\mathrm{P}_{\mathrm{i}, \mathrm{t}}$ and $\mathrm{P}_{\mathrm{i}, \mathrm{t}-\mathrm{1}}$ are the closing values of index $\mathrm{i}$ on the days $\mathrm{t}$ and $\mathrm{t}-1$, respectively.

The accuracy of GARCH models could be affected by spurios regressions. In order to avoid them we analyze the stationarity of returns by employing the classic Augmented Dickey - Fuller (ADF) tests (Dickey \& Fuller, 1979). For all indexes, the returns graphical representations suggest the use of intercept terms in the ADF regressions. We employ Akaike (1973) Information Criteria to choose the numbers of lags for ADF regressions. We also analyze the autocorrelation and the heteroscedasticity on returns using ARMA (p, q) models, in which the values of $p$ and $q$ are determined by Box-Jenkins methodology (Box et al., 1994). We investigate the ARCH effects on the residuals of these regressions by employing the Ljung Box test Q and the Engle Lagrange Multiplier (LM) test (Ljung \& Box, 1978; Engle, 1982).

We reveal the turn-of-the-month effects using a dummy variable (TOM) which takes value 1 for days from the TOM periods and 0 for the other days. There are many points of view about the range of TOM period. In our investigation we follow Lakonishok and Smidt (1988) approach which include in TOM period the first three days of a month and the last day of the previous month.

A GARCH model is described by two main equations: the conditional mean and the conditional variance. In the first one, we introduce the TOM dummy variable to reveal the seasonality of returns $\left(\mathrm{r}_{\mathrm{t}}\right)$ :

$$
r_{t}=\mu_{0}+\mu_{1} \times T O M_{t}+\sum_{k=1}^{n}\left(\xi_{k} \times r_{t-k}\right)+\varepsilon_{t}
$$

where:

- $\mu_{0}$ is a constant reflecting the returns of the days not included in TOM period;

- $\mu_{1}$ is a coefficient which reflects the differences between the returns from the days of TOM period and those from the rest of the month;

$-\xi_{\mathrm{k}}$ is a coefficient of the k-order lagged returns;

- $\mathrm{n}$ represents the number of lagged returns, which is calculated by the Akaike Final Prediction Error Criterion (Akaike, 1969);

- $\varepsilon_{\mathrm{t}}$ is the error term. 
The equation of the returns conditional variance $\left(\sigma_{t}^{2}\right)$ has different forms for the three GARCH models. For the (Engle, 1982; Bollersev, 1986) standard form we include the TOM dummy variable to reveal the seasonality of the stock returns volatility:

$$
\sigma_{t}^{2}=\omega+v \times T O M_{t}+\sum_{k=1}^{q} \alpha_{k} \times \varepsilon_{t-k}^{2}+\sum_{l=1}^{p}\left(\beta_{l} \times \sigma_{t-l}^{2}\right)
$$

where:

- $\omega$ is a constant term reflecting the volatility of the returns from the days not included in TOM period;

$-v$ is a coefficient which reflects the TOM effects on the stocks volatility;

$-\alpha_{\mathrm{k}}(\mathrm{k}=1,2, \ldots \mathrm{q})$ are the coefficients associated to the squared values of the lagged values of error term from the conditional mean equation;

- $\mathrm{q}$ is the number of lagged values of the error term, which is calculated by the Akaike Information Criteria (Akaike, 1973);

- $\beta_{\mathrm{l}}(\mathrm{l}=1,2, \ldots \mathrm{p})$ are coefficients associated to the lagged values of the conditional variance;

- $\mathrm{p}$ is the number of lagged values of conditional variance, calculated also by the Akaike Information Criteria.

For the (Glosten et al. 1993) GJR GARCH model, the conditional variance of the returns contains the TOM dummy variable to express the turn-of-the-month effects on the volatility:

$$
\sigma_{t}^{2}=\omega+v \times T O M_{t}+\sum_{k=1}^{q}\left[\alpha_{k} \times \varepsilon_{t-k}^{2}+\gamma_{k} \times \varepsilon_{t-k}^{2} \times I\left(\varepsilon_{t-k}<0\right)\right]+\sum_{l=1}^{p}\left(\beta_{l} \times \sigma_{t-l}^{2}\right)
$$

where:

- $\mathrm{I}\left(\varepsilon_{\mathrm{t}-\mathrm{k}}<0\right)$ is a dummy variable, taking the value 1 if the $\mathrm{k}$-lagged error term is strict negative and value zero otherwise;

- $\gamma_{\mathrm{k}}$ is the coefficient associated to the variable $\mathrm{I}\left(\varepsilon_{\mathrm{t}-\mathrm{k}}<0\right)$, expressing the asymmetrical responses of the volatility on the good and bad news.

For the Nelson (1991) EGARCH model, we include the TOM dummy variable in the conditional variance equation which reflects the volatility of returns:

$$
\ln \left(\sigma_{t}^{2}\right)=\varpi+v \times \text { TOM }_{t}+\sum_{j=1}^{p} \beta_{j} \times \ln \left(\sigma_{t-j}^{2}\right)+\sum_{k=1}^{p}\left[\gamma_{k} \times \frac{\varepsilon_{t-k}}{\sqrt{\sigma_{t-k}^{2}}}+\alpha_{k} \times\left(\frac{\varepsilon_{t-k}}{\sqrt{\sigma_{t-k}^{2}}}-\sqrt{\frac{2}{\pi}}\right)\right]
$$

which could be transformed in:

$$
\ln \left(\sigma_{t}^{2}\right)=\omega+v \times \operatorname{TOM}_{t}+\sum_{j=1}^{p} \beta_{j} \times \ln \left(\sigma_{t-j}^{2}\right)+\sum_{k=1}^{p}\left[\gamma_{k} \times \varepsilon_{t-k}+\alpha_{k} \times\left|\varepsilon_{t-k}\right|\right]
$$

where $\omega=\varpi-\sqrt{\frac{2}{\pi}} \times \sum_{k=1}^{p} \alpha_{k}$

After performing the regressions of GARCH models we analyze the presence of ARCH effects on their residuals by employing Lagrange Multiplier (LM) tests. A model is validated only if it eliminates ARCH effects. For each return, we choose between the valid models employing as criteria the specific GARCH terms significance.

\section{Empirical Results}

The results of ADF tests on the indexes returns are presented in the Table 2. For both subsamples, the null hypothesis of unit root was rejected for all 32 returns. The Table 3 reports the results of Ljung - Box Q and ARCH LM tests on the residuals of ARMA models which indicate, for both sub-samples 
International Conference "Risk in Contemporary Economy"

ISSN online 2344-5386 ISSN print 2067-0532

XIVth Edition, 2013, Galati, Romania,

"Dunarea de Jos" University of Galati - Faculty of Economics and Business Administration

and for all 32 returns, that the null hypothesis of autocorrelation and the heteroscedasticity of the residuals cannot be rejected.

The GARCH models with TOM dummy variables applied to the indexes of advanced markets indicate some forms of turn-of-the -month seasonality. For the first sub-sample, the results of the conditional mean equation, which are presented in the Table 4, reveal significant positive TOM effects for the returns of 14 indexes: AEX General, ATX, BEL-20, CAC 40, DAX, FTSE 100, Hang Seng, FTSE MIB, Nikkei 225, OSEAX, S\&P TSX Composite, Straits Times, SSMI and TAIEX. The Table 5 reports the results of the conditional variance equation for the same period of time. From all the returns, only those from All Ordinaries index displayed a negative TOM effect on the volatility. For the second sub-sample, the coefficients of the conditional mean equation, which are presented in the Table 6, indicate significant TOM effects for the returns of three indexes: ATX, BEL-20 and TAIEX. For the same period of time, the returns of two indexes, Hang Seng and OSEAX displayed positive TOM effects on the volatility (Table 7).

We continue by performing GARCH models with TOM dummy variables for the indexes of emerging markets. The results of the conditional mean equation for the second sub-sample, which are presented in the Table 8, reveal significant positive TOM effects for the returns of ten indexes: Athex Composite Share, BET-C, Bovespa, BSE 30, BUX, IDX Composite, IPC, KOSPI, SSE Composite and TA 100. For the same period of time, returns of eight indexes displayed TOM effects on the volatility: BET-C, BUX, IDX Composite, IPC, KLSE, OMXT, SEMDEX and SSE Composite (Table 9). For the second sub-sample, the coefficients of the conditional mean equation indicate positive TOM effects for the returns of 13 indexes: Athex Composite Share, BET-C, Bovespa, BSE 30, BUX, CROBEX, IDX Composite, KLSE Composite, IPC, KOSPI, OMXT, PX and TA 100 (Table 10). The results of the conditional variance equation, presented in the Table 11, reveal no TOM effect on volatility for any of the emerging markets indexes.

For all GARCH regressions, we perform ARCH LM tests on their residuals. The results, presented in the Table 12, indicate no remaining ARCH effects.

\section{Conclusions}

In this paper we approached the persistence in time of TOM effects from advanced and emerging markets. We found, for both categories of markets, that passing from a relative quiet period of time to a turbulent one induced significant changes.

For the indexes from the advanced markets, the turbulences provoked a significant decline of TOM effects in returns. From 2000 to 200614 indexed displayed this calendar anomaly on their returns. However, for 11 of them TOM effects in returns disappeared between 2007 and 2013. The changes from TOM effects on volatility were also significant. During the quiet period we found this seasonality for a single index. This TOM effect disappeared during the turbulent period, being replaced by this seasonality of two other indexes.

The changes induced by the turbulences to TOM effects of returns from emerging markets were different to those from developed markets. Between 2000 and 2006 the returns of ten indexes displayed this seasonality. From 2007 to 2013 TOM effect on returns of one from those indexes disappeared, but it was replaced by the other three indexes returns seasonality. Instead, TOM effects on volatility, which was found for eight indexes between 2000 and 2006, disappeared from 2007 to 2013.

We could offer two main explanations for the differences between advanced and emerging markets regarding the persistence in time of TOM effects on returns. The first is that seasonality follows the Dimson and Marsh (1999) Murphy's law for calendar anomalies, but on different stages for the advanced and emerging markets. The advanced markets passed in a stage of TOM effects decline, while the emerging are still in an incipient stage. Other explanation could refer to the different impact of turbulences on the risk perceptions from advanced and emerging markets. For the advanced markets this impact was substantial since they were perceived mainly as having moderate risks. This major change of risk perceptions led to different behaviours of the investors causing the decline of calendar anomalies. Instead, for the emerging markets the impact of turbulence was less consistent since most of them were perceived already as having high risks.

The results of GARCH models suggest that TOM effects on volatility are not persistent in time for both advanced and emerging markets. This investigation could be extended to other advanced and emerging markets.

\section{References}

1. Agrawal, A. and Tandon, K. (1994), "Anomalies or illusions?, Evidence from stock markets in eighteen countries", Journal of International Money and Finance, 13, pp. 83-106.

2. Akaike, H. (1969), "Fitting autoregressive models for prediction", Annals of the Institute of Statistical Mathematics 21, pp. $243-247$. 
International Conference "Risk in Contemporary Economy"

ISSN online 2344-5386 ISSN print 2067-0532

XIVth Edition, 2013, Galati, Romania,

"Dunarea de Jos" University of Galati - Faculty of Economics and Business Administration

3. Akaike, H. (1973), "Information theory and an extension of the maximum likelihood principle", in B. Petrov and F. Csáki (eds), 2nd International Symposium on Information Theory, Académiai Kiadó, Budapest, pp. 267-281.

4. Akaike, H. (1974), "A new look at the statistical model identification", IEEE Transactions on Automatic Control AC-19, pp. 716-723.

5. Ariel, Robert A. (1987), "A Monthly Effect in Stock Returns", Journal of Financial Economics, Vol. 18, pp. 161-74.

6. Ball, R. and Kothari, S.P. (1991), "Security Returns Around Earnings Announcements", The Accounting Review, Vol. 66, (October), pp. 718-738.

7. Bollerslev, T. (1986), "Generalized Autoregressive Conditional Heteroskedasticity", Journal of Econometrics, No. 3, pp. 307-327.

8. Booth, T., Kallunki, J-P. and Martikainen T. (2001), "Liquidity and the turn-of-the-month effect: Evidence from Finland", Journal of International Financial Markets, Institutions and Money, 11, pp. 137-146.

9. Box, G. E. P, Jenkins, G. M. and Reinsel, G. C. (1994), Time Series Analysis, Forecasting and Control, 3rd ed. Prentice Hall, Englewood Clifs, NJ

10. Brown, P., D.B. Keim, A.W. Keleidon and Marsh, T.A. (1983), "Stock Return Seasonalities and the Tax-Loss-Selling-Hypothesis: Analysis of the Arguments and Australian Evidence", Journal of Financial Economics, Vol. 12, pp.105-127.

11. Cadsby, Charles B., Mitchell Ratner (1992), "Turn-of-month and pre-holiday effects on stock returns: Some international evidence", Journal of Banking and Finance 16, pp. 497-509.

12. Compton, W. (2000), "The Evolving Turn-Of-The-Month Effect: Evidence from Pacific Rim Countries", University of North CarolinaWilmington, Working Papers.

13. Desai, Jay and Trivedi, Arti (2012), "A Survey of Day of the Month Effect in World Stock Markets" (November 6), Available at SSRN: http://ssrn.com/abstract $=2171634$

14. Dickey, D. A. and Fuller, W. A. (1979), "Estimators for autoregressive time series with a unit root", Journal of the American Statistical Association 74, pp. 427-431.

15. Dimson, E. and Marsh, P. (1999), "Murphy's law and market anomalies", Journal of Portfolio Management, 25, pp. 53-69.

16. Dumitriu Ramona, Stefanescu Razvan, Nistor Costel (2011), "Analysis Of Within - Month Effects On The Bucharest Stock Exchange", The 17th International Conference The Knowledge-Based Organization, organized by "Nicolae Bălcescu" Land Forces Academy Sibiu, 24-26 November

17. Dumitriu, Ramona, Stefanescu, Razvan and Nistor, Costel (2012), "The Halloween effect during quiet and turbulent times", The 18th International Conference "The Knowledge-Based Organization", Sibiu, 2012 - Conference Proceedings

18. Dumitriu, Ramona and Stefanescu, Razvan (2013), DOW effects in returns and in volatility of stock markets during quiet and turbulent times, Proceedings of the 5th International Conference on Economics and Administration No. 2013 (22. May 2013): pp. 143-169.

19. Dzhabarov, C. and Ziemba, W.T. (2010), “Do Seasonal Anomalies Still Work?", Journal of Portfolio Management, 36 (3), pp. 93-104.

20. Ederington, L. H. \& Lee, J. H. (1993), "How markets process information: News releases and volatility", Journal of Finance, 48:4, pp. 1161-1191.

21. Engle, R.F. (1982), "Autoregressive Conditional Heteroscedasticity with Estimates of the Variance of United Kingdom Inflation", Econometrica, No. 50, pp. 987-1007.

22. Fama, E.F. (1970), "Efficient capital markets: a review of theory and empirical work", Journal of Finance, No. 25, pp. 383-441.

23. Fama, F. E. (1991), "Efficient Capital Markets: II", Journal of Finance, vol. 46, No. 5, pp. 1575-1617.

24. Fama, E.F. (1998), "Market Efficiency, Long-Term Returns and Behavioural Finance", Journal of Financial Economics, 49, pp. 283306.

25. Fama, Eugene F. and French, Kenneth R. (2007), "Dissecting Anomalies", CRSP Working Paper No. 610, Available at SSRN: http://ssrn.com/abstract $=911960$

26. Glosten, L. R., Jagannathan, R. and Runkle, D.E. (1993), "On the Relation between the Expected Value and the Volatility of the Nominal Excess Returns on Stocks", Journal of Finance, Vol. 48 No. 5, pp. 1779-1801.

27. Graham, Michael, Nikkinen Jussi and Sahlström Petri (2003), "Relative importance of scheduled macroeconomic news for stock market investors", Journal of Economics and Finance, Springer, vol. 27(2), pp. 153-165, June.

28. Gultekin, M.N. and Gultekin, N.B. (1983), "Stock Market Seasonality: International Evidence", Journal of Financial Economics, Vol.12, pp. 469-481.

29. Haugen, R A. and Lakonishok, J. (1993), "The Incredible January Effect: The Stock Market's Unsolved Mystery", Dow Jones-Irwin, Homewood, Ill.

30. Hensel, C. R., Sick, G. A. and Ziemba, William T. (1995), "The turn-of-the-month effect in the futures markets, 1982-1992", Review of Futures Markets, 8, pp. 827-856.

31. Hensel, C.R. and Ziemba, W.T. (1996), "Investment Results from Exploiting Turn-of-the-Month Effects:

32. Should You Pay Attention to the Turn of the Month?", Journal of Portfolio Management, 22 (3) pp. 17-23.

33. Jacobs, B.I. and Levy, K.N. (1988), "Calendar Anomalies: Abnormal Returns at Calendar Turning Points", Financial Analyst Journal, 44 (6) pp. 28-39.

34. Jensen, Michael C. (1978), "Some Anomalous Evidence Regarding Market Efficiency", Journal of Financial Economics 6, pp. 95-101.

35. Joshi, Nayan Krishna and Fatta Bahadur K.C. (2005), "The Nepalese Stock Market: Efficiency and Calendar Anomalies", Economic Review, Vol. 17, No. 17, Available at SSRN: http://ssrn.com/abstract=743666

36. Kohers T. and Patel, J.B. (1999), "A New Time of the Month Anomaly in Stock Index Returns", Applied Economics Letters, 6(2), pp. 115-120.

37. Kunkel R. A., Compton W. S., Beyer S. (2003), "The turn-of-the-month effect still lives: the international evidence", International Review of Financial Analysis 137, pp. 1-15.

38. Kunkel R. A., Compton W. S. (1998), "A Tax-Free Exploitation of the Turn-of-the-Month Effect: CREF", Financial Services Review 7(1), pp. 11-23.

39. Lakonishok, J. and Smidt, S. (1988), "Are Seasonal Anomalies Real? A Ninety Years Perspective", Review of Financial Studies, 1(4), pp. 403-425.

40. Lakonishok J., Schleifer A., Thaler R., Vishny R. (1991), "Window Dressing by Pension Fund Managers", The American Economic Review, 82, pp. 227-232.

41. Li, K., Sarkar, A. and Wang, Z. (2003), "Diversification Benefits of Emerging Markets Subject to Portfolio Constraints", Journal of Empirical Finance, 10, pp. 57-80.

42. Liu, Lan (2013), "The Turn-Of-The-Month Effect in the S\&P 500 (2001-2011)", Journal of Business \& Economics Research - June 2013 Volume 11, Number 6. 
International Conference "Risk in Contemporary Economy"

ISSN online 2344-5386 ISSN print 2067-0532

XIVth Edition, 2013, Galati, Romania,

"Dunarea de Jos" University of Galati - Faculty of Economics and Business Administration

43. Ljung, G. and Box, G. (1978), "On a Measure of Lack of Fit in Time Series Models", Biometrika, 65, pp. 297-303.

44. Marquering, W., Nisser, J. and Valla, T. (2006), "Disappearing anomalies: A dynamic analysis of the persistence of anomalies", Applied Financial Economics, 16, pp. 291-302.

45. Martikainen, T., J. Perttunen and W.T. Ziemba (1994), "The turn-of-the-month effect in the world's stock markets", January 1988January 1990, Financial Markets and Portfolio Management

46. McGuinness, P. B. (2006), "Turn-of-the-month return effects for small cap Hong Kong stocks", Applied EconomicsLetters, 13, pp. 891-898.

47. Mills, T.C., Coutts, J.A. (1995), "Calendar Effects in the London Stock Exchange FTSE Indices", European Journal of Finance, no. 1, pp.79-93.

48. Nelson, D. B. (1991), "Conditional Heteroskedasticity In Asset Returns: A New Approach", Econometrica, No. 59, pp. 347-370

49. Nikkinen, Jussi and Sahlstrom, Petri (2004), "Scheduled domestic and US macroeconomic news and stock valuation in Europe", Journal of Multinational Financial Management, Elsevier, vol. 14(3), pp. 201-215, July.

50. Nikkinen, J., Sahlström, P. and Äijö, J. (2007), "Turn-of-the-month and intramonth effects: Explanation from the important macroeconomic news announcements", Journal of Futures markets, 27, pp. 105-126.

51. Nikkinen, Jussi, Sahlstrom, Petri and Takko, Karri (2009), "Turn-of-the-month and Intramonth Anomalies and U.S. Macroeconomic News Announcements on the Thinly Traded Finnish Stock Market", International Journal of Economics and Finance, Vol. 1, No. 2.

52. Ogden, J.P. (1987), "The End of the Month as a Preferred Habitat: A Test of Operational Efficiency in the Money Market", Journal of Financial and Quantitative Analysis, 22 (3) pp. 329-344.

53. Ogden, Joseph P. (1990), "Turn-of-month evaluations of liquid profits and stock returns: A common explanation for the monthly and January effects", Journal of Finance 45(4), pp. 1259-1272.

54. Penman, Stephen H. (1987), "The distribution of earnings news over time and seasonalities in aggregate stock returns", Journal of Financial Economics, Volume 18, Issue 2, June 1987, pp. 199-228.

55. Peterson D.R. (1990), "Stock return seasonalities and earnings information", Journal of Quantitative Analysis, 25, pp. 74-86.

56. Pettengill, G. and Jordan, B. (1988), "A comprehensive examination of volume effects and seasonality in daily security returns", Journal of Financial Research, 11, pp. 57-70.

57. Phylaktis, K. and Ravazzolo, F. (2002), "Measuring financial and economic integration with equity prices in emerging markets", Journal of International Money and Finance, 21, pp. 879-903.

58. Reschenhofer, Erhard (2010), "Further Evidence on the Turn-of-the-Month Effect", Business and Economics Journal, Volume 2010: BEJ-16.

59. Ritter, J.R. and Chopra, N. (1989), "Portfolio Rebalancing and the Turn of the Year Effect", Journal of Finance 44, pp. 149-166.

60. Schweret, G.W. (2002), "Anomalies and Market Efficiency", NBER Working Paper No. 9277

61. Stefanescu Razvan, Dumitriu Ramona (2011), "Turn - Of - The - Month Effect On The Bucharest Stock Exchange", Proceedings of The International Conference on Economics and Administration ICEA - FAA 2011.

62. Szakmary, A. and Kiefer, D. (2004), "The disappearing January/turn of the year effect: Evidence from stock index futures and cash markets, Journal of Futures Markets, 24, pp. 756-784.

63. Thaler, Richard H. (1987), "Anomalies: Weekend, Holiday, Turn of the Month, and Intraday Effects", Journal of Economic Perspectives, 1(2), pp. 169-177.

64. Westerfield, R. and Keim, D. and Jaffe, J. (1989), "Earnings yields, market values, and stock returns", The Journal of Finance 44(1), pp. 135-148.

65. Wiley, Jonathan and Zumpano, Leonard V. (2009), "Institutional Investment and the Turn of the Month Effect: Evidence from REITs" (July 15), Journal of Real Estate Finance and Economics, Vol. 39, No. 2, 2009, Available at SSRN: http://ssrn.com/abstract $=1434582$

66. Wong, K.A. (1995), "Is There An Intra-Month Effect On Stock Returns In Developing Stock Markets?", Applied Financial Economics, 5, pp. 285-289.

67. Xu, Wei and McConnell, John J. (2006), "Equity Returns at the Turn of the Month", Available at SSRN: http://ssrn.com/abstract $=917884$

68. Ziemba, William, T. (1991), "Japanese security market regularities: monthly, turn-of-the-month and year, holiday and Golden Week effects", Japan and the World Economy 3, pp. 119-146.

\section{Appendix}

Table 1 - Indexes from advanced and emerging markets used in TOM effects investigation

\begin{tabular}{|c|c|c|}
\hline \multicolumn{1}{|c|}{ Index } & Market & Source of data \\
\hline \multicolumn{2}{|c|}{ Panel A: advanced markets } \\
\hline AEX General & Amsterdam Stock Exchange & http://finance.yahoo.com \\
\hline All Ordinaries & Australian Securities Exchange & http://finance.yahoo.com \\
\hline ATX & Vienna Stock Exchange & http://finance.yahoo.com \\
\hline BEL-20 & Brussels Stock Exchange & http://finance.yahoo.com \\
\hline CAC 40 & Paris Bourse & http://finance.yahoo.com \\
\hline DAX & Frankfurt Stock Exchange & http://finance.yahoo.com \\
\hline FTSE 100 & London Stock Exchange & http://finance.yahoo.com \\
\hline Hang Seng & Hong Kong Stock Exchange & http://finance.yahoo.com \\
\hline FTSE MIB & Borsa Milano & http://www.stockrageous.com/ \\
\hline Nikkei 225 & Tokyo Stock Exchange & http://finance.yahoo.com \\
\hline
\end{tabular}


International Conference "Risk in Contemporary Economy"

ISSN online 2344-5386 ISSN print 2067-0532

XIVth Edition, 2013, Galati, Romania,

"Dunarea de Jos" University of Galati - Faculty of Economics and Business Administration

\begin{tabular}{|c|c|c|}
\hline OSEAX & Oslo Stock Exchange & http://finance.yahoo.com \\
\hline $\begin{array}{c}\text { S\&P TSX } \\
\text { Composite }\end{array}$ & Toronto Stock Exchange & http://finance.yahoo.com \\
\hline Standard \& Poor's & New York Stock Exchange & http://finance.yahoo.com \\
\hline Straits Times & Singapore Exchange & http://finance.yahoo.com \\
\hline SSMI & SIX Swiss Exchange & http://finance.yahoo.com \\
\hline TAIEX & Taiwan Stock Exchange & http://finance.yahoo.com \\
\hline \multicolumn{3}{|c|}{ Panel B: emerging markets } \\
\hline $\begin{array}{c}\text { Athex Composite } \\
\text { Share }\end{array}$ & Athens Stock Exchange & http://finance.yahoo.com \\
\hline BET-C & Bucharest Stock Exchange & http://www.bvb.ro \\
\hline Bovespa & $\begin{array}{c}\text { São Paulo Stock, Mercantile \& } \\
\text { Futures Exchange }\end{array}$ & http://finance.yahoo.com \\
\hline BSE 30 & Bombay Stock Exchange & http://finance.yahoo.com \\
\hline BUX & Budapest Stock Exchange & http://bse.hu \\
\hline CROBEX & Zagreb Stock Exchange & http://www.zse.hr \\
\hline IDX Composite & Indonesia Stock Exchange & http://finance.yahoo.com \\
\hline IPC & Mexican Stock Exchange & http://finance.yahoo.com \\
\hline KLSE Composite & Kuala Lumpur Stock Exchange & http://finance.yahoo.com \\
\hline KOSPI & Korea Stock Exchange & http://finance.yahoo.com \\
\hline MerVal & Buenos Aires Stock Exchange & http://finance.yahoo.com \\
\hline OMXT & Talinn Stock Exchange & http://www.nasdaqomxbaltic.com \\
\hline $\mathrm{PX}$ & Prague Stock Exchange & http://www.pse.cz/ \\
\hline SEMDEX & The Stock Exchange of Mauritius & $\begin{array}{c}\text { http://www.stockexchangeofmauritius.co } \\
\mathrm{m}\end{array}$ \\
\hline SSE Composite & Shanghai Stock Exchange & http://finance.yahoo.com \\
\hline TA 100 & Tel Aviv Stock Exchange & http://www.tase.co.il \\
\hline
\end{tabular}

Table 2 - Results of ADF tests for the returns

\begin{tabular}{|c|c|c|c|c|}
\hline \multirow{2}{*}{ Index } & \multicolumn{2}{|c|}{ First sub-sample } & \multicolumn{2}{|c|}{ Second sub-sample } \\
\hline & Number of lags & Test statistics & Number of lags & Test statistics \\
\hline \multicolumn{5}{|c|}{ Panel A: advanced markets } \\
\hline AEX General & 18 & $-10.7644^{* * *}$ & 18 & $-9.07468 * * *$ \\
\hline All Ordinaries & 20 & $-9.67463^{* * *}$ & 23 & $-8.70532^{* * *}$ \\
\hline ATX & 13 & $-9.88074^{* * *}$ & 24 & $-6.70246^{* * *}$ \\
\hline BEL-20 & 15 & $-11.0221^{* * *}$ & 24 & $-7.61911^{* * *}$ \\
\hline CAC 40 & 18 & $-10.2277^{* * *}$ & 24 & $-8.21157^{* * *}$ \\
\hline DAX & 23 & $-8.24136^{* * *}$ & 24 & $-8.07536^{* * *}$ \\
\hline FTSE 100 & 21 & $-9.82347^{* * *}$ & 17 & $-10.532^{* * *}$ \\
\hline Hang Seng & 16 & $-9.67231^{* * *}$ & 18 & $-8.95955^{* * *}$ \\
\hline FTSE MIB & 14 & $-10.282^{* * *}$ & 16 & $-8.79638^{* * *}$ \\
\hline Nikkei 225 & 23 & $-8.36668^{* * *}$ & 20 & $-9.11076^{* * *}$ \\
\hline OSEAX & 24 & $-7.11064^{* * *}$ & 16 & $-8.76572^{* * *}$ \\
\hline $\begin{array}{c}\text { S\&P TSX } \\
\text { Composite }\end{array}$ & 24 & $-7.89807^{* * *}$ & 12 & $-11.8436^{* * *}$ \\
\hline $\begin{array}{c}\text { Standard \& } \\
\text { Poor's }\end{array}$ & 18 & $-8.96899^{* * *}$ & 20 & $-9.35182^{* * *}$ \\
\hline Straits Times & 12 & $-8.41722^{* * *}$ & 21 & $-7.72856^{* * *}$ \\
\hline SSMI & 19 & $-9.30069 * * *$ & 14 & $-11.7669^{* * *}$ \\
\hline TAIEX & 21 & $-8.63978^{* * *}$ & 19 & $-7.46551^{* * *}$ \\
\hline \multicolumn{5}{|c|}{ Panel B: emerging markets } \\
\hline
\end{tabular}


"Dunarea de Jos" University of Galati - Faculty of Economics and Business Administration

\begin{tabular}{|c|c|c|c|c|}
\hline $\begin{array}{c}\text { Athex } \\
\text { Composite } \\
\text { Share }\end{array}$ & 20 & $-9.85546^{* * *}$ & 16 & $-8.66939^{* * *}$ \\
\hline BET-C & 19 & $-8.15408^{* * *}$ & 21 & $-7.2736^{* * *}$ \\
\hline Bovespa & 15 & $-8.80295^{* * *}$ & 19 & $-8.48072^{* * *}$ \\
\hline BSE 30 & 16 & $-9.22486^{* * *}$ & 16 & $-8.60657^{* * *}$ \\
\hline BUX & 21 & $-8.32031^{* * *}$ & 24 & $-6.94695^{* * *}$ \\
\hline CROBEX & 21 & $-9.1649^{* * *}$ & 20 & $-7.86167^{* * *}$ \\
\hline IDX Composite & 19 & $-8.0831^{* * *}$ & 24 & $-6.7728^{* * *}$ \\
\hline IPC & 10 & $-12.7359^{* * *}$ & 16 & $-9.79851^{* * *}$ \\
\hline KLSE & 24 & $-7.67952^{* * *}$ & 13 & $-10.4009^{* * *}$ \\
\hline Composite & 23 & $-7.46765^{* * *}$ & 21 & $-9.14872^{* * *}$ \\
\hline KOSPI & 24 & $-8.56384^{* * *}$ & 24 & $-7.10717^{* * *}$ \\
\hline MerVal & 21 & $-7.87358^{* * *}$ & 24 & $-7.97929^{* * *}$ \\
\hline OMXT & 16 & $-9.09127^{* * *}$ & 17 & $-8.37226^{* * *}$ \\
\hline PX & 23 & $-6.56819^{* * *}$ & 18 & $-7.54523^{* * *}$ \\
\hline SEMDEX & 20 & $-8.21549^{* * *}$ & 24 & $-7.7843^{* * *}$ \\
\hline SSE Composite & 14 & $-0.881075^{* * *}$ & 23 & $-6.56284^{* * *}$ \\
\hline TA 100 & & & \\
\hline
\end{tabular}

Note: *** means significant at 0.01 level.

Table 3 - Results of Ljung-Box Q Tests and ARCH LM Tests on the residuals of ARMA models

\begin{tabular}{|c|c|c|c|c|}
\hline \multirow{2}{*}{ Index } & \multicolumn{2}{|c|}{ First sub-sample } & \multicolumn{2}{|c|}{ Second sub-sample } \\
\hline & $\begin{array}{c}\text { Ljung-Box Q } \\
\text { Test }\end{array}$ & ARCH LM Test & Ljung-Box Q Test & ARCH LM Test \\
\hline \multicolumn{5}{|c|}{ Panel A: advanced markets } \\
\hline AEX General & $\begin{array}{c}13.448 \\
\left\{0.0041^{* * *}\right\}\end{array}$ & $\begin{array}{c}388.302 \\
\left\{0.0001^{* * *}\right\}\end{array}$ & $\begin{array}{c}8.974 \\
\left\{0.0307^{* *}\right\}\end{array}$ & $\begin{array}{c}384.611 \\
\left\{0.0001^{* * *}\right\}\end{array}$ \\
\hline All Ordinaries & $\begin{array}{c}6.492 \\
\left\{0.0892^{*}\right\}\end{array}$ & $\begin{array}{c}89.124 \\
\left\{0.0001^{* * *}\right\}\end{array}$ & $\begin{array}{c}5.841 \\
\{0.082 *\}\end{array}$ & $\begin{array}{c}216.041 \\
\left\{0.0001^{* * *}\right\}\end{array}$ \\
\hline ATX & $\begin{array}{c}28.014 \\
\left\{0.064^{*}\right\}\end{array}$ & $\begin{array}{c}183.048 \\
\left\{0.0001^{* * *}\right\}\end{array}$ & $\begin{array}{c}59.175 \\
\left\{0.0004^{* * *}\right\}\end{array}$ & $\begin{array}{c}285.571 \\
\left\{0.0001^{* * *}\right\}\end{array}$ \\
\hline BEL-20 & $\begin{array}{c}16.124 \\
\left\{0.0017^{* * *}\right\}\end{array}$ & $\begin{array}{c}257.841 \\
\left\{0.0001^{* * *}\right\}\end{array}$ & $\begin{array}{c}28.473 \\
\left\{0.0147^{* *}\right\}\end{array}$ & $\begin{array}{c}241.685 \\
\left\{0.0001^{* * *}\right\}\end{array}$ \\
\hline CAC 40 & $\begin{array}{c}6.384 \\
\left\{0.0946^{*}\right\}\end{array}$ & $\begin{array}{c}287.706 \\
\left\{0.0001^{* * *}\right\}\end{array}$ & $\begin{array}{c}15.784 \\
\left\{0.0014^{* * *}\right\}\end{array}$ & $\begin{array}{c}250.803 \\
\left\{0.0001^{* * *}\right\}\end{array}$ \\
\hline DAX & $\begin{array}{c}28.002 \\
\left\{0.0001^{* * *}\right\}\end{array}$ & $\begin{array}{c}374.284 \\
\left\{0.0001^{* * *}\right\}\end{array}$ & $\begin{array}{c}16.011 \\
\left\{0.0019^{* * *}\right\}\end{array}$ & $\begin{array}{c}205.476 \\
\left\{0.0001^{* * *}\right\}\end{array}$ \\
\hline FTSE 100 & $\begin{array}{c}14.519 \\
\left\{0.0028^{* * *}\right\}\end{array}$ & $\begin{array}{c}388.663 \\
\left\{0.0001^{* * *}\right\}\end{array}$ & $\begin{array}{c}28.249 \\
\left\{0.0001^{* * *}\right\}\end{array}$ & $\begin{array}{c}312.741 \\
\left\{0.0001^{* * *}\right\}\end{array}$ \\
\hline Hang Seng & $\begin{array}{c}9.341 \\
\left\{0.0238^{* *}\right\}\end{array}$ & $\begin{array}{c}55.608 \\
\left\{0.0001^{* * *}\right\}\end{array}$ & $\begin{array}{c}17.731 \\
\left\{0.0242^{* *}\right\}\end{array}$ & $\begin{array}{c}328.062 \\
\left\{0.0001^{* * *}\right\}\end{array}$ \\
\hline FTSE MIB & $\begin{array}{c}4.287 \\
\left\{0.0884^{*}\right\}\end{array}$ & $\begin{array}{c}87.028 \\
\left\{0.0001^{* * *}\right\}\end{array}$ & $\begin{array}{c}8.609 \\
\left\{0.0461^{* *}\right\}\end{array}$ & $\begin{array}{c}299.15 \\
\left\{0.0001^{* * *}\right\}\end{array}$ \\
\hline Nikkei 225 & $\begin{array}{c}3.2055 \\
\left\{0.0847^{*}\right\}\end{array}$ & $\begin{array}{c}88.2094 \\
\left\{0.0001^{* * *}\right\}\end{array}$ & $\begin{array}{c}29.416 \\
\left\{0.0645^{*}\right\} \\
\end{array}$ & $\begin{array}{c}408.017 \\
\left\{0.0001^{* * *}\right\}\end{array}$ \\
\hline OSEAX & $\begin{array}{c}20.0419 \\
\left\{0.0741^{*}\right\}\end{array}$ & $\begin{array}{c}242.831 \\
\left\{0.0001^{* * *}\right\}\end{array}$ & $\begin{array}{c}23.087 \\
\left\{0.0205^{* *}\right\} \\
\end{array}$ & $\begin{array}{c}382.551 \\
\left\{0.0001^{* * *}\right\}\end{array}$ \\
\hline $\begin{array}{c}\text { S\&P TSX } \\
\text { Composite }\end{array}$ & $\begin{array}{c}25.841 \\
\left\{0.0898^{*}\right\}\end{array}$ & $\begin{array}{c}67.037 \\
\left\{0.0001^{* * *}\right\}\end{array}$ & $\begin{array}{c}18.105 \\
\left\{0.0004^{* * *}\right\}\end{array}$ & $\begin{array}{c}375.016 \\
\left\{0.0001^{* * *}\right\}\end{array}$ \\
\hline $\begin{array}{c}\text { Standard \& } \\
\text { Poor's }\end{array}$ & $\begin{array}{c}25.0114 \\
\left\{0.0471^{* *}\right\}\end{array}$ & $\begin{array}{c}233.644 \\
\left\{0.0001^{* * *}\right\}\end{array}$ & $\begin{array}{c}8.284 \\
\left\{0.0437^{* *}\right\}\end{array}$ & $\begin{array}{c}386.124 \\
\left\{0.0001^{* * *}\right\}\end{array}$ \\
\hline
\end{tabular}


International Conference "Risk in Contemporary Economy"

ISSN online 2344-5386 ISSN print 2067-0532

XIVth Edition, 2013, Galati, Romania,

"Dunarea de Jos" University of Galati - Faculty of Economics and Business Administration

\begin{tabular}{|c|c|c|c|c|}
\hline Straits Times & $\begin{array}{c}12.017 \\
\left\{0.0071^{* * *}\right\}\end{array}$ & $\begin{array}{c}51.4197 \\
\left\{0.0001^{* * *}\right\}\end{array}$ & $\begin{array}{c}31.873 \\
\left\{0.0008^{* * *}\right\}\end{array}$ & $\begin{array}{c}251.861 \\
\left\{0.0001^{* * *}\right\}\end{array}$ \\
\hline SSMI & $\begin{array}{c}12.480 \\
\left\{0.0069^{* * *}\right\}\end{array}$ & $\begin{array}{c}301.841 \\
\left\{0.0001^{* * *}\right\}\end{array}$ & $\begin{array}{c}41.523 \\
\left\{0.0001^{* * *}\right\}\end{array}$ & $\begin{array}{c}382.011 \\
\left\{0.0001^{* * *}\right\}\end{array}$ \\
\hline TAIEX & $\begin{array}{c}10.192 \\
\left\{0.0168^{* *}\right\}\end{array}$ & $\begin{array}{c}144.203 \\
\left\{0.0001^{* * *}\right\}\end{array}$ & $\begin{array}{c}17.371 \\
\left\{0.0242^{* *}\right\} \\
\end{array}$ & $\begin{array}{c}129.466 \\
\left\{0.0001^{* * *}\right\}\end{array}$ \\
\hline \multicolumn{5}{|c|}{ Panel B: emerging markets } \\
\hline $\begin{array}{c}\text { Athex } \\
\text { Composite } \\
\text { Share } \\
\end{array}$ & $\begin{array}{c}12.157 \\
\left\{0.0006^{* * *}\right\}\end{array}$ & $\begin{array}{c}265.031 \\
\left\{0.0001^{* * *}\right\}\end{array}$ & $\begin{array}{c}17.483 \\
\left\{0.079^{*}\right\}\end{array}$ & $\begin{array}{c}84.017 \\
\left\{0.0001^{* * *}\right\}\end{array}$ \\
\hline BET-C & $\begin{array}{c}7.650 \\
\left\{0.0568^{*}\right\}\end{array}$ & $\begin{array}{c}171.070 \\
\left\{0.0001^{* * *}\right\}\end{array}$ & $\begin{array}{c}7.924 \\
\left\{0.073^{*}\right\}\end{array}$ & $\begin{array}{c}256.780 \\
\left\{0.0001^{* * *}\right\}\end{array}$ \\
\hline Bovespa & $\begin{array}{c}73.016 \\
\{0.0749 *\}\end{array}$ & $\begin{array}{c}69.1004 \\
\left\{0.0001^{* * *}\right\}\end{array}$ & $\begin{array}{c}41.617 \\
\left\{0.0033^{* * *}\right\}\end{array}$ & $\begin{array}{c}386.407 \\
\left\{0.0001^{* * *}\right\}\end{array}$ \\
\hline BSE 30 & $\begin{array}{c}14.064 \\
\left\{0.0032^{* * *}\right\} \\
\end{array}$ & $\begin{array}{c}337.161 \\
\left\{0.0001^{* * *}\right\}\end{array}$ & $\begin{array}{c}24.914 \\
\left\{0.0482^{* *}\right\} \\
\end{array}$ & $\begin{array}{c}115.198 \\
\left\{0.0001^{* * *}\right\}\end{array}$ \\
\hline BUX & $\begin{array}{c}8.002 \\
\left\{0.0416^{* *}\right\} \\
\end{array}$ & $\begin{array}{c}55.8782 \\
\left\{0.0001^{* * *}\right\} \\
\end{array}$ & $\begin{array}{c}25.416 \\
\left\{0.0002^{* * *}\right\} \\
\end{array}$ & $\begin{array}{c}205.017 \\
\left\{0.0001^{* * *}\right\} \\
\end{array}$ \\
\hline CROBEX & $\begin{array}{c}55.167 \\
\left\{0.0717^{*}\right\} \\
\end{array}$ & $\begin{array}{c}133.406 \\
\left\{0.0001^{* * *}\right\}\end{array}$ & $\begin{array}{c}8.061 \\
\left\{0.0415^{* *}\right\} \\
\end{array}$ & $\begin{array}{c}317.773 \\
\left\{0.0001^{* * *}\right\} \\
\end{array}$ \\
\hline $\begin{array}{c}\text { IDX } \\
\text { Composite }\end{array}$ & $\begin{array}{c}4.391 \\
\left\{0.0374^{* *}\right\}\end{array}$ & $\begin{array}{c}60.8274 \\
\left\{0.0001^{* * *}\right\}\end{array}$ & $\begin{array}{c}6.731 \\
\left\{0.0428^{* *}\right\}\end{array}$ & $\begin{array}{c}168.257 \\
\left\{0.0001^{* * *}\right\}\end{array}$ \\
\hline IPC & $\begin{array}{c}47.785 \\
\left\{0.0253^{* *}\right\}\end{array}$ & $\begin{array}{c}128.768 \\
\left\{0.0001^{* * *}\right\} \\
\end{array}$ & $\begin{array}{c}14.683 \\
\left\{0.0285^{* *}\right\}\end{array}$ & $\begin{array}{c}194.758 \\
\left\{0.0001^{* * *}\right\} \\
\end{array}$ \\
\hline $\begin{array}{c}\text { KLSE } \\
\text { Composite }\end{array}$ & $\begin{array}{c}24.011 \\
\left\{0.0628^{*}\right\}\end{array}$ & $\begin{array}{c}161.740 \\
\left\{0.0001^{* * *}\right\}\end{array}$ & $\begin{array}{c}27.061 \\
\left\{0.0283^{* *}\right\}\end{array}$ & $\begin{array}{c}306.937 \\
\left\{0.0001^{* * *}\right\}\end{array}$ \\
\hline KOSPI & $\begin{array}{c}30.014 \\
\left\{0.0284^{* *}\right\}\end{array}$ & $\begin{array}{c}94.374 \\
\left\{0.0001^{* * *}\right\}\end{array}$ & $\begin{array}{c}50.164 \\
\left\{0.0906^{*}\right\} \\
\end{array}$ & $\begin{array}{c}321.684 \\
\left\{0.0001^{* *}\right\}\end{array}$ \\
\hline MerVal & $\begin{array}{c}38.876 \\
\left\{0.0282^{* *}\right\}\end{array}$ & $\begin{array}{c}265.862 \\
\left\{0.0001^{* * *}\right\}\end{array}$ & $\begin{array}{c}11.068 \\
\left\{0.0071^{* * *}\right\}\end{array}$ & $\begin{array}{c}248.146 \\
\left\{0.0001^{* * *}\right\}\end{array}$ \\
\hline OMXT & $\begin{array}{c}59.740 \\
\left\{0.0569^{*}\right\} \\
\end{array}$ & $\begin{array}{c}38.551 \\
\left\{0.0001^{* * *}\right\}\end{array}$ & $\begin{array}{c}11.408 \\
\left\{0.0206^{* *}\right\}\end{array}$ & $\begin{array}{c}103.175 \\
\left\{0.0001^{* *}\right\}\end{array}$ \\
\hline PX & $\begin{array}{c}42.169 \\
\left\{0.0841^{*}\right\} \\
\end{array}$ & $\begin{array}{c}1323.668 \\
\left\{0.0001^{* * *}\right\}\end{array}$ & $\begin{array}{c}6.128 \\
\left\{0.0461^{* *}\right\}\end{array}$ & $\begin{array}{c}321.429 \\
\left\{0.0001^{* * *}\right\}\end{array}$ \\
\hline SEMDEX & $\begin{array}{c}7.374 \\
\{0.0622 *\}\end{array}$ & $\begin{array}{c}107.301 \\
\left\{0.0001^{* * *}\right\}\end{array}$ & $\begin{array}{c}6.288 \\
\left\{0.0874^{*}\right\}\end{array}$ & $\begin{array}{c}294.705 \\
\left\{0.0001^{* * *}\right\}\end{array}$ \\
\hline $\begin{array}{c}\text { SSE } \\
\text { Composite }\end{array}$ & $\begin{array}{c}53.046 \\
\{0.0794 *\} \\
\end{array}$ & $\begin{array}{c}34.8006 \\
\left\{0.0001^{* * *}\right\}\end{array}$ & $\begin{array}{c}9.649 \\
\left\{0.0074^{*}\right\}\end{array}$ & $\begin{array}{c}62.486 \\
\left\{0.0001^{* * *}\right\}\end{array}$ \\
\hline TA 100 & $\begin{array}{c}7.9048 \\
\left\{0.0492^{* *}\right\}\end{array}$ & $\begin{array}{c}62.8041 \\
\left\{0.0001^{* * *}\right\}\end{array}$ & $\begin{array}{c}29.714 \\
\left\{0.0943^{*}\right\} \\
\end{array}$ & $\begin{array}{c}167.518 \\
\left\{0.0001^{* * *}\right\}\end{array}$ \\
\hline
\end{tabular}

Notes: p-values are within accolades; ${ }^{* * *}, * *, *$; mean significant at $0.01,0.05$, and 0.1 levels, respectively.

Table 4 - Conditional mean equation for the returns of advanced markets indexes from the first sub-sample

\begin{tabular}{|c|c|c|c|}
\hline Index & Constant term & $\begin{array}{c}\text { Coefficient of TOM } \\
\text { dummy variable }\end{array}$ & First order lagged returns \\
\hline AEX General & -0.0222559 & 0.116456 & $\mathrm{x}$ \\
& $(0.0237487)$ & {$[0.0565602)$} & \\
\hline All Ordinaries & {$[-0.9371]$} & {$[2.059]^{* *}$} & $\mathrm{x}$ \\
& 0.0417625 & 0.0491725 & \\
& {$[0.0159982)$} & {$[0.0485281)$} & $\mathrm{x}$ \\
& {$[2.610]^{* * *}$} & {$[1.013]$} & \\
\hline ATX & 0.0545373 & 0.175671 & 0.0439425 \\
& {$[0.021593)$} & {$[0.0530207)$} & \\
\hline BEL-20 & {$[2.526]^{* *}$} & {$[3.313]^{* * *}$} & 0.128121 \\
\hline
\end{tabular}


"Dunarea de Jos" University of Galati - Faculty of Economics and Business Administration

\begin{tabular}{|c|c|c|c|}
\hline & $\begin{array}{c}(0.0184115) \\
{[1.607]}\end{array}$ & $\begin{array}{c}(0.0503765) \\
{[2.543]^{* *}} \\
\end{array}$ & $\begin{array}{c}(0.0242438) \\
{[1.813]^{*}} \\
\end{array}$ \\
\hline CAC 40 & $\begin{array}{c}-0.0113106 \\
(0.0250143) \\
{[-0.4522]}\end{array}$ & $\begin{array}{c}0.140274 \\
(0.0523767) \\
{[2.678]^{* * *}}\end{array}$ & $\begin{array}{c}-0.0487905 \\
(0.0253502) \\
{[-1.925]^{*}}\end{array}$ \\
\hline DAX & $\begin{array}{c}-0.0136522 \\
(0.0263685) \\
{[-0.5177]}\end{array}$ & $\begin{array}{c}0.176902 \\
(0.0637447) \\
{[2.775]^{* * *}} \\
\end{array}$ & $\begin{array}{c}-0.0429036 \\
(0.0234809) \\
{[-1.827]^{*}} \\
\end{array}$ \\
\hline FTSE 100 & $\begin{array}{c}-0.000012 \\
(0.019761) \\
{[-0.0024]}\end{array}$ & $\begin{array}{c}0.185410 \\
(0.0492036) \\
{[3.768]^{* * *}}\end{array}$ & $\begin{array}{c}-0.0891438 \\
(0.0233277) \\
{[-3.821]^{* * *}}\end{array}$ \\
\hline Hang Seng & $\begin{array}{c}0.00971395 \\
(0.0288676) \\
{[0.3365]}\end{array}$ & $\begin{array}{c}0.150271 \\
(0.062548) \\
{[2.402]^{* *}}\end{array}$ & $\mathrm{x}$ \\
\hline FTSE MIB & $\begin{array}{c}-0.00168589 \\
(0.0218604) \\
{[-0.07712]}\end{array}$ & $\begin{array}{c}0.102520 \\
(0.0477724) \\
{[2.146]^{*}}\end{array}$ & $\mathrm{x}$ \\
\hline Nikkei 225 & $\begin{array}{c}-0.0173986 \\
(0.0252829) \\
{[-0.6882]}\end{array}$ & $\begin{array}{c}0.179677 \\
(0.0647276) \\
{[2.776]^{* * *}} \\
\end{array}$ & $\mathrm{x}$ \\
\hline OSEAX & $\begin{array}{c}0.0633505 \\
(0.0273017) \\
{[2.320]^{* *}} \\
\end{array}$ & $\begin{array}{c}0.205407 \\
(0.0587306) \\
{[3.497]^{* * *}}\end{array}$ & $\mathrm{x}$ \\
\hline $\begin{array}{c}\text { S\&P TSX } \\
\text { Composite }\end{array}$ & $\begin{array}{c}0.0165995 \\
(0.000011) \\
{[919.7]^{* * *}}\end{array}$ & $\begin{array}{c}0.153008 \\
(0.0003699) \\
{[413.6]^{* * *}}\end{array}$ & $\begin{array}{c}0.0168208 \\
(0.000014) \\
{[693.7]^{* * *}}\end{array}$ \\
\hline Standard \& Poor's & $\begin{array}{c}0.00014403 \\
(0.0225252) \\
{[0.0064]}\end{array}$ & $\begin{array}{c}0.0547802 \\
(0.0518975) \\
{[1.056]}\end{array}$ & $\begin{array}{c}-0.0567926 \\
(0.0240624) \\
{[-2.360]^{* *}}\end{array}$ \\
\hline Straits Times & $\begin{array}{c}-0.00280729 \\
(0.0224764) \\
{[-0.1249]}\end{array}$ & $\begin{array}{c}0.226430 \\
(0.0471757) \\
{[4.80]^{* * *}} \\
\end{array}$ & $\mathrm{x}$ \\
\hline SSMI & $\begin{array}{c}-0.00566719 \\
(0.0205401) \\
{[-0.2759]}\end{array}$ & $\begin{array}{c}0.128116 \\
(0.0479468) \\
{[2.672]^{* * *}}\end{array}$ & $\mathrm{x}$ \\
\hline TAIEX & $\begin{array}{c}-0.0229975 \\
(0.0413377) \\
{[-0.5563]}\end{array}$ & $\begin{array}{c}0.313040 \\
(0.0796324) \\
{[3.931]^{* * *}}\end{array}$ & $\mathrm{x}$ \\
\hline
\end{tabular}

Notes: Standard errors in round brackets; z-statistics in square brackets; ${ }^{* * *},{ }^{* *},{ }^{*}$ mean significant at 0.01 , 0.05 , and 0.1 levels, respectively.

Table 5 - Conditional variance equation for the returns of advanced markets indexes from the first sub-sample

\begin{tabular}{|c|c|c|c|c|c|}
\hline Index & Constant term & $\begin{array}{c}\text { Coefficient of } \\
\text { TOM dummy } \\
\text { variable }\end{array}$ & alpha & gamma & beta \\
\hline AEX General & 0.0110108 & 0.0178953 & 0.0343692 & 0.136416 & 0.919410 \\
GJR GARCH (1,1) & {$[0.0059404)$} & {$[0.0254532)$} & $(0.0054637)$ & {$[0.0216266)$} & {$[0.0115834)$} \\
& {$[1.854]^{*}$} & {$[0.7031]$} & {$[6.290]^{* * *}$} & {$[6.308]^{* * *}$} & {$[79.37]^{* * *}$} \\
\hline All Ordinaries & 0.0122718 & -0.0217885 & 0.0752859 & $\mathrm{X}$ & 0.907428 \\
GARCH (1,1) & {$[0.0030896)$} & {$[0.00858916)$} & $(0.0123626)$ & & {$[0.0142595)$} \\
& {$[3.972]^{* * *}$} & {$[-2.537]^{* *}$} & {$[6.090]^{* * *}$} & & {$[63.64]^{* * *}$} \\
\hline ATX & -0.146360 & 0.0339447 & 0.161733 & -0.0740930 & 0.939804 \\
EGARCH $(1,1)$ & {$[0.0295689)$} & $(0.0383671)$ & $(0.0320675)$ & $(0.0192904)$ & $(0.0187596)$ \\
& {$[-4.950]^{* * *}$} & {$[0.8847]$} & {$[5.044]^{* * *}$} & {$[-3.841]^{* * *}$} & {$[50.10]^{* * *}$} \\
\hline BEL-20 & -0.164363 & -0.0107943 & 0.201481 & -0.0976767 & 0.977649 \\
\hline
\end{tabular}


"Dunarea de Jos" University of Galati - Faculty of Economics and Business Administration

\begin{tabular}{|c|c|c|c|c|c|}
\hline EGARCH $(1,1)$ & $\begin{array}{c}(0.0264583) \\
{[-6.212]^{* * *}} \\
\end{array}$ & $\begin{array}{c}(0.0180311) \\
{[-0.5986]}\end{array}$ & $\begin{array}{c}(0.033462) \\
{[6.021]^{* * *}}\end{array}$ & $\begin{array}{c}(0.0169808) \\
{[5.752]^{* * *}}\end{array}$ & $\begin{array}{c}(0.00559443) \\
{[174.8]^{* * *}}\end{array}$ \\
\hline $\begin{array}{c}\text { CAC } 40 \\
\text { EGARCH }(1,1)\end{array}$ & $\begin{array}{c}-0.0796203 \\
(0.0156708) \\
{[-5.081]^{* * *}}\end{array}$ & $\begin{array}{c}0.0252850 \\
(0.0362548) \\
{[0.6974]}\end{array}$ & $\begin{array}{c}0.0963412 \\
(0.0179016) \\
{[5.382]^{* * *}}\end{array}$ & $\begin{array}{c}-0.113840 \\
(0.0142675) \\
{[-7.979]^{* * *}}\end{array}$ & $\begin{array}{c}0.985805 \\
(0.00273819) \\
{[360.0]^{* * *}}\end{array}$ \\
\hline $\begin{array}{c}\text { DAX } \\
\text { EGARCH }(1,1)\end{array}$ & $\begin{array}{c}-0.0924866 \\
(0.0140718) \\
{[-6.572]^{* * *}}\end{array}$ & $\begin{array}{c}0.0241156 \\
(0.0377292) \\
{[0.6392]}\end{array}$ & $\begin{array}{c}0.118154 \\
(0.0156768) \\
{[7.537]^{* * *}}\end{array}$ & $\begin{array}{c}-0.110523 \\
(0.0152848) \\
{[-7.231]^{* * *}}\end{array}$ & $\begin{array}{c}0.983516 \\
(0.00357591) \\
{[275.0]^{* * *}}\end{array}$ \\
\hline $\begin{array}{c}\text { FTSE } 100 \\
\text { GARCH }(1,1)\end{array}$ & $\begin{array}{c}0.00848004 \\
(0.00423610) \\
{[2.002]^{* *}}\end{array}$ & $\begin{array}{c}0.00802300 \\
(0.0196789) \\
{[0.407]}\end{array}$ & $\begin{array}{c}0.0971542 \\
(0.0142747) \\
{[6.806]^{* * *}}\end{array}$ & $\mathrm{x}$ & $\begin{array}{c}0.893019 \\
(0.015420) \\
{[57.91]^{* * *}}\end{array}$ \\
\hline $\begin{array}{c}\text { Hang Seng } \\
\text { EGARCH }(1,1)\end{array}$ & $\begin{array}{c}-0.0683421 \\
(0.0146201) \\
{[-4.675]^{* * *}}\end{array}$ & $\begin{array}{c}0.0464948 \\
(0.0444245) \\
{[1.047]} \\
\end{array}$ & $\begin{array}{c}0.0806308 \\
(0.014625) \\
{[5.513]^{* * *}} \\
\end{array}$ & $\begin{array}{c}-0.0591354 \\
(0.0146082) \\
{[-4.048]^{* * *}}\end{array}$ & $\begin{array}{c}0.988332 \\
(0.00369394) \\
{[267.6]^{* * *}} \\
\end{array}$ \\
\hline $\begin{array}{c}\text { FTSE MIB } \\
\text { EGARCH }(1,1)\end{array}$ & $\begin{array}{c}-0.0984247 \\
(0.0158588) \\
{[-6.206]^{* * *}}\end{array}$ & $\begin{array}{c}0.0520276 \\
(0.0342290) \\
{[1.520]}\end{array}$ & $\begin{array}{c}0.110968 \\
(0.0187955) \\
{[5.904]^{* * *}}\end{array}$ & $\begin{array}{c}-0.106833 \\
(0.0170447) \\
{[-6.268]^{* * *}}\end{array}$ & $\begin{array}{c}0.986487 \\
(0.00371607) \\
{[265.5]^{* * *}}\end{array}$ \\
\hline $\begin{array}{c}\text { Nikkei } 225 \\
\text { EGARCH }(1,1)\end{array}$ & $\begin{array}{c}-0.111609 \\
(0.0165689) \\
{[-6.736]^{* * *}}\end{array}$ & $\begin{array}{c}0.0281719 \\
(0.0326641) \\
{[0.8625]} \\
\end{array}$ & $\begin{array}{c}0.151601 \\
(0.0207677) \\
{[7.300]^{* * *}}\end{array}$ & $\begin{array}{c}-0.0698402 \\
(0.0203019) \\
{[-3.440]^{* * *}}\end{array}$ & $\begin{array}{c}0.975052 \\
(0.00799656) \\
{[121.9]^{* * *}}\end{array}$ \\
\hline $\begin{array}{c}\text { OSEAX } \\
\text { GARCH }(1,1)\end{array}$ & $\begin{array}{c}0.0704072 \\
(0.0284268) \\
{[2.477]^{* *}}\end{array}$ & $\begin{array}{c}0.0402941 \\
(0.0365026) \\
{[1.104]}\end{array}$ & $\begin{array}{c}0.107278 \\
(0.0249421) \\
{[4.301]^{* * *}}\end{array}$ & $\mathrm{x}$ & $\begin{array}{c}0.829894 \\
(0.0406741) \\
{[20.402]^{* * *}}\end{array}$ \\
\hline $\begin{array}{c}\text { S\&P TSX } \\
\text { Composite } \\
\text { EGARCH }(1,1) \\
\end{array}$ & $\begin{array}{c}-0.100740 \\
(0.0191964) \\
{[-5.248]^{* * *}}\end{array}$ & $\begin{array}{c}-0.00376700 \\
(0.0397529) \\
{[-0.09476]} \\
\end{array}$ & $\begin{array}{c}0.121959 \\
(0.0220408) \\
{[5.533]^{* * *}}\end{array}$ & $\begin{array}{c}-0.0629678 \\
(0.0146888) \\
{[-4.287]^{* * *}}\end{array}$ & $\begin{array}{c}0.983810 \\
(0.00510750) \\
{[192.6]^{* * *}}\end{array}$ \\
\hline $\begin{array}{l}\text { Standard \& Poor's } \\
\text { GJR GARCH }(1,1)\end{array}$ & $\begin{array}{c}0.00389552 \\
(0.0045579) \\
{[0.8547]} \\
\end{array}$ & $\begin{array}{c}0.0159927 \\
(0.0200660) \\
{[0.7970]} \\
\end{array}$ & $\begin{array}{c}0.0264042 \\
(0.0052171) \\
{[5.061]^{* * *}} \\
\end{array}$ & $\begin{array}{c}0.983795 \\
(0.0176236) \\
{[55.82]^{* * *}} \\
\end{array}$ & $\begin{array}{c}0.939482 \\
(0.0115747) \\
{[81.17]^{* * *}} \\
\end{array}$ \\
\hline $\begin{array}{c}\text { Straits Times } \\
\text { GJR GARCH }(1,1)\end{array}$ & $\begin{array}{c}0.00683276 \\
(0.00773663) \\
{[0.8832]} \\
\end{array}$ & $\begin{array}{c}0.0381963 \\
(0.025520) \\
{[1.497]} \\
\end{array}$ & $\begin{array}{c}0.0460894 \\
(0.0160305) \\
{[2.875]^{* * *}}\end{array}$ & $\begin{array}{c}0.0564102 \\
(0.0225836) \\
{[2.498]^{* * *}}\end{array}$ & $\begin{array}{c}0.913367 \\
(0.0220984) \\
{[41.33]^{* * *}}\end{array}$ \\
\hline $\begin{array}{c}\text { SSMI } \\
\text { GJR GARCH }(1,1)\end{array}$ & $\begin{array}{c}0.0122984 \\
(0.0049636) \\
{[2.478]^{* *}} \\
\end{array}$ & $\begin{array}{c}0.0226376 \\
(0.0208049) \\
{[1.088]} \\
\end{array}$ & $\begin{array}{c}0.0400058 \\
(0.0067224) \\
{[5.951]^{* * *}} \\
\end{array}$ & $\begin{array}{c}0.987979 \\
(0.0438083) \\
{[22.55]^{* * *}} \\
\end{array}$ & $\begin{array}{c}0.903927 \\
(0.0137512) \\
{[65.73]^{* * *}} \\
\end{array}$ \\
\hline $\begin{array}{c}\text { TAIEX } \\
\text { EGARCH }(1,1)\end{array}$ & $\begin{array}{c}-0.0858264 \\
(0.0169583) \\
{[-5.061]^{* * *}} \\
\end{array}$ & $\begin{array}{c}0.0554853 \\
(0.0411640) \\
{[1.348]} \\
\end{array}$ & $\begin{array}{c}0.103382 \\
(0.0218031) \\
{[4.742]^{* * *}} \\
\end{array}$ & $\begin{array}{c}-0.0511987 \\
(0.0127985) \\
{[-4.000]^{* * *}}\end{array}$ & $\begin{array}{c}0.991497 \\
(0.00377399) \\
{[262.7]^{* * *}} \\
\end{array}$ \\
\hline
\end{tabular}

Notes: Standard errors in round brackets; z-statistics in square brackets; ${ }^{* * *}, * *, *$ mean significant at 0.01 , 0.05 , and 0.1 levels, respectively.

Table 6 - Conditional mean equation for the returns of advanced markets indexes from the second sub-sample

\begin{tabular}{|c|c|c|c|}
\hline Index & Constant term & $\begin{array}{c}\text { Coefficient of TOM } \\
\text { dummy variable }\end{array}$ & $\begin{array}{c}\text { First order lagged } \\
\text { returns }\end{array}$ \\
\hline AEX General & -0.0157314 & 0.0525904 & $\mathrm{x}$ \\
& {$[0.0221046)$} & $(0.0600496)$ & $\mathrm{X}$ \\
\hline All Ordinaries & 0.0162120 & 0.00784815 & $\mathrm{X}$ \\
& {$[0.0218783)$} & $(0.0615830)$ & \\
\hline ATX & {$[0.7410]$} & {$[0.1274]$} & $\mathrm{x}$ \\
\hline BEL-20 & 0.00761101 & 0.136464 & \\
& {$[0.0355879)$} & {$[0.0814578)$} & {$[1.675]^{*}$} \\
\hline
\end{tabular}


"Dunarea de Jos" University of Galati - Faculty of Economics and Business Administration

\begin{tabular}{|c|c|c|c|}
\hline & {$[-1.650]^{*}$} & {$[1.723]^{*}$} & \\
\hline CAC 40 & $\begin{array}{c}-0.0344582 \\
(0.0330332) \\
{[-1.043]} \\
\end{array}$ & $\begin{array}{c}0.0887529 \\
(0.0788549) \\
{[1.126]} \\
\end{array}$ & $\begin{array}{c}-0.0448172 \\
(0.0225427) \\
{[-1.988]^{* *}} \\
\end{array}$ \\
\hline DAX & $\begin{array}{c}0.0122723 \\
(0.0475976) \\
{[0.2578]} \\
\end{array}$ & $\begin{array}{c}0.0513080 \\
(0.0661754) \\
{[0.7753]}\end{array}$ & $\mathrm{x}$ \\
\hline FTSE 100 & $\begin{array}{c}-0.0140183 \\
(0.0278866) \\
{[-0.5027]}\end{array}$ & $\begin{array}{c}0.103009 \\
(0.0796944) \\
{[1.293]} \\
\end{array}$ & $\begin{array}{c}-0.0461126 \\
(0.0232440) \\
{[-1.984]^{* *}} \\
\end{array}$ \\
\hline Hang Seng & $\begin{array}{c}0.0113496 \\
(0.0331520) \\
{[0.3424]} \\
\end{array}$ & $\begin{array}{c}0.126788 \\
(0.0812942) \\
{[1.560]} \\
\end{array}$ & $\mathrm{x}$ \\
\hline FTSE MIB & $\begin{array}{c}-0.0769714 \\
(0.0979201) \\
{[-0.7861]}\end{array}$ & $\begin{array}{c}0.0887786 \\
(0.376346) \\
{[0.2359]}\end{array}$ & $\mathrm{x}$ \\
\hline Nikkei 225 & $\begin{array}{c}0.00948411 \\
(0.0346326) \\
{[0.2738]} \\
\end{array}$ & $\begin{array}{c}-0.0413383 \\
(0.0895086) \\
{[-0.4618]} \\
\end{array}$ & $\begin{array}{c}-0.0489348 \\
(0.0237261) \\
{[-2.062]^{* *}} \\
\end{array}$ \\
\hline OSEAX & $\begin{array}{c}0.0169618 \\
(0.0176401) \\
{[0.9615]} \\
\end{array}$ & $\begin{array}{c}0.0460600 \\
(0.0529289) \\
{[0.8702]}\end{array}$ & $\mathrm{x}$ \\
\hline S\&P TSX Composite & $\begin{array}{c}0.00343087 \\
(0.00674625) \\
{[0.5086]} \\
\end{array}$ & $\begin{array}{c}0.0223866 \\
(0.0790002) \\
0.2834]\end{array}$ & $\mathrm{x}$ \\
\hline Standard \& Poor's & $\begin{array}{c}0.0209382 \\
(0.0186565) \\
{[1.122]} \\
\end{array}$ & $\begin{array}{c}0.0443056 \\
(0.0405277) \\
{[1.093]}\end{array}$ & $\begin{array}{c}-0.0804564 \\
(0.0219635) \\
{[-3.663]^{* *}}\end{array}$ \\
\hline Straits Times & $\begin{array}{c}0.00311705 \\
(0.00036151) \\
{[8.622]^{* * *}}\end{array}$ & $\begin{array}{c}0.0593810 \\
(0.0454762) \\
{[1.306]}\end{array}$ & $\mathrm{x}$ \\
\hline SSMI & $\begin{array}{c}-0.0160259 \\
(0.0207381) \\
{[-0.7728]}\end{array}$ & $\begin{array}{c}0.0838886 \\
(0.0537225) \\
{[1.562]}\end{array}$ & $\mathrm{x}$ \\
\hline TAIEX & $\begin{array}{c}-0.0124721 \\
(0.000028) \\
{[-1103]^{* * *}}\end{array}$ & $\begin{array}{c}0.159869 \\
(0.0552382) \\
{[2.894]^{* * *}}\end{array}$ & $\mathrm{x}$ \\
\hline
\end{tabular}

Notes: Standard errors in round brackets; z-statistics in square brackets; ***, **, * mean significant at 0.01 , 0.05 , and 0.1 levels, respectively.

Table 7 - Conditional variance equation for the returns of advanced markets indexes from the second subsample

\begin{tabular}{|c|c|c|c|c|c|}
\hline Index & Constant term & $\begin{array}{c}\text { Coefficient of } \\
\text { TOM dummy } \\
\text { variable }\end{array}$ & alpha & gamma & beta \\
\hline $\begin{array}{c}\text { AEX General } \\
\text { EGARCH } \\
(1,1)\end{array}$ & $\begin{array}{c}-0.0988652 \\
(0.0207209) \\
{[-4.771]^{* * *}}\end{array}$ & $\begin{array}{c}0.0320727 \\
(0.04099) \\
{[0.7823]} \\
\end{array}$ & $\begin{array}{c}0.125118 \\
(0.0246505) \\
{[5.076]^{* * *}} \\
\end{array}$ & $\begin{array}{c}-0.151706 \\
(0.0200167) \\
{[-7.579]^{* * *}} \\
\end{array}$ & $\begin{array}{c}0.983943 \\
(0.00537226) \\
{[183.2]^{* * *}} \\
\end{array}$ \\
\hline $\begin{array}{c}\text { All } \\
\text { Ordinaries } \\
\text { EGARCH } \\
(1,1)\end{array}$ & $\begin{array}{c}-0.129645 \\
(0.0171387) \\
{[-7.564]^{* * *}}\end{array}$ & $\begin{array}{c}0.0527250 \\
(0.03318) \\
{[1.589]}\end{array}$ & $\begin{array}{c}0.152837 \\
(0.0213890) \\
{[7.146]^{* * *}}\end{array}$ & $\begin{array}{c}-0.121653 \\
(0.0179967) \\
{[-6.760]^{* * *}}\end{array}$ & $\begin{array}{c}0.971915 \\
(0.00694945) \\
{[139.9]^{* * *}}\end{array}$ \\
\hline $\begin{array}{c}\text { ATX } \\
\text { GARCH }(1,1)\end{array}$ & $\begin{array}{c}0.0256162 \\
(0.0170105) \\
{[1.506]}\end{array}$ & $\begin{array}{c}0.0441676 \\
(0.05839) \\
{[0.7564]}\end{array}$ & $\begin{array}{c}0.0904280 \\
(0.0196968) \\
{[4.591]^{* * *}}\end{array}$ & $\mathrm{x}$ & $\begin{array}{c}0.899566 \\
(0.021012) 8 \\
{[42.81]^{* * *}}\end{array}$ \\
\hline BEL-20 & -0.117777 & 0.0019901 & 0.166299 & -0.160937 & 0.968790 \\
\hline
\end{tabular}


“Dunarea de Jos” University of Galati - Faculty of Economics and Business Administration

\begin{tabular}{|c|c|c|c|c|c|}
\hline $\begin{array}{c}\text { EGARCH } \\
(1,1) \\
\end{array}$ & $\begin{array}{c}(0.0240737) \\
{[-4.892]^{*}}\end{array}$ & $\begin{array}{l}(0.03956) \\
{[0.05030]}\end{array}$ & $\begin{array}{c}(0.0289074) \\
{[5.753]^{* * *}}\end{array}$ & $\begin{array}{c}(0.0250578) \\
{[-6.423]^{* * *}}\end{array}$ & $\begin{array}{c}(0.00906285) \\
{[106.9]^{* * *}}\end{array}$ \\
\hline $\begin{array}{c}\text { CAC } 40 \\
\text { EGARCH } \\
(1,1)\end{array}$ & $\begin{array}{c}-0.0753279 \\
(0.0188667) \\
{[-3.993]^{* * *}}\end{array}$ & $\begin{array}{c}0.0093966 \\
(0.03939) \\
{[0.2386]}\end{array}$ & $\begin{array}{c}0.118802 \\
(0.0207761) \\
{[5.718]^{* * *}}\end{array}$ & $\begin{array}{c}-0.193742 \\
(0.0286075) \\
{[-6.772]^{* * *}}\end{array}$ & $\begin{array}{c}0.968521 \\
(0.00904658) \\
{[107.1]^{* * *}}\end{array}$ \\
\hline $\begin{array}{c}\text { DAX } \\
\text { EGARCH } \\
(1,1) \\
\end{array}$ & $\begin{array}{c}-0.086995 \\
(0.034526) \\
{[-2.520]^{* *}}\end{array}$ & $\begin{array}{c}-0.020411 \\
(0.09941) \\
{[0.2053]}\end{array}$ & $\begin{array}{c}0.133006 \\
(0.022602) \\
{[5.884]^{* * *}}\end{array}$ & $\begin{array}{c}-0.156354 \\
(0.0252676) \\
{[-6.188]^{* * *}}\end{array}$ & $\begin{array}{c}0.977006 \\
(0.00803671) \\
{[121.6]^{* * *}}\end{array}$ \\
\hline $\begin{array}{c}\text { FTSE } 100 \\
\text { EGARCH } \\
(1,1)\end{array}$ & $\begin{array}{c}-0.0990395 \\
(0.0190664) \\
{[-5.194]^{* * *}}\end{array}$ & $\begin{array}{c}-0.011637 \\
(0.0325) \\
0.3580]\end{array}$ & $\begin{array}{c}0.135870 \\
(0.0233632) \\
{[5.816]^{* * *}}\end{array}$ & $\begin{array}{c}-0.138166 \\
(0.0198014) \\
{[-6.978]^{* * *}}\end{array}$ & $\begin{array}{c}0.976717 \\
(0.00697736) \\
{[140.0]^{* * *}}\end{array}$ \\
\hline $\begin{array}{c}\text { Hang Seng } \\
\text { GARCH }(1,1)\end{array}$ & $\begin{array}{c}-0.00394120 \\
(0.0132849) \\
{[-0.2967]}\end{array}$ & $\begin{array}{c}0.124840 \\
(0.05948) \\
{[2.099]^{* *}} \\
\end{array}$ & $\begin{array}{c}0.0750389 \\
(0.012628) \\
{[5.942]^{* * *}} \\
\end{array}$ & $\mathrm{x}$ & $\begin{array}{c}0.918502 \\
(0.0129583) \\
{[70.88]^{* * *}}\end{array}$ \\
\hline $\begin{array}{c}\text { FTSE MIB } \\
\text { EGARCH } \\
(1,1) \\
\end{array}$ & $\begin{array}{c}-0.0865575 \\
(0.0706533) \\
{[-1.225]}\end{array}$ & $\begin{array}{c}0.0151308 \\
(0.39959) \\
{[0.03787]}\end{array}$ & $\begin{array}{c}0.125623 \\
(0.0244258) \\
{[5.143]^{* * *}}\end{array}$ & $\begin{array}{c}-0.103834 \\
(0.0157874) \\
{[-6.577]^{* * *}}\end{array}$ & $\begin{array}{c}0.985366 \\
(0.00543891) \\
{[181.2]^{* * *}}\end{array}$ \\
\hline $\begin{array}{c}\text { Nikkei } 225 \\
\text { EGARCH } \\
(1,1) \\
\end{array}$ & $\begin{array}{c}-0.132996 \\
(0.0208238) \\
{[-6.387]^{* * *}}\end{array}$ & $\begin{array}{c}0.0351508 \\
(0.03842) \\
{[0.9148]}\end{array}$ & $\begin{array}{c}0.194626 \\
(0.0280226) \\
{[6.945]^{* * *}} \\
\end{array}$ & $\begin{array}{c}-0.0907503 \\
(0.0220602) \\
{[-4.114]^{* * *}}\end{array}$ & $\begin{array}{c}0.965870 \\
(0.00935738) \\
{[103.2]^{* * *}}\end{array}$ \\
\hline $\begin{array}{c}\text { OSEAX } \\
\text { EGARCH } \\
(1,1) \\
\end{array}$ & $\begin{array}{c}-0.118296 \\
(0.0199315) \\
{[-5.935]^{* * *}}\end{array}$ & $\begin{array}{c}0.0640045 \\
(0.03714) \\
{[1.723]^{*}} \\
\end{array}$ & $\begin{array}{c}0.142915 \\
(0.0242996) \\
{[5.881]^{* * *}} \\
\end{array}$ & $\begin{array}{c}-0.100726 \\
(0.0160765) \\
{[-6.265]^{* * *}}\end{array}$ & $\begin{array}{c}0.987014 \\
(0.00403649) \\
{[244.5]^{* * *}} \\
\end{array}$ \\
\hline $\begin{array}{c}\text { S\&P TSX } \\
\text { Composite } \\
\text { EGARCH } \\
(1,1)\end{array}$ & $\begin{array}{c}-0.109189 \\
(0.0176127) \\
{[-6.199]^{* * *}}\end{array}$ & $\begin{array}{c}0.0528085 \\
(0.05039) \\
{[1.048]}\end{array}$ & $\begin{array}{c}0.125513 \\
(0.0196740) \\
{[6.380]^{* * *}}\end{array}$ & $\begin{array}{c}-0.118723 \\
(0.0177372) \\
{[-6.693]^{* * *}}\end{array}$ & $\begin{array}{c}0.986915 \\
(0.00391724) \\
{[251.9]^{* * *}}\end{array}$ \\
\hline $\begin{array}{c}\text { Standard \& } \\
\text { Poor's } \\
\text { EGARCH } \\
(1,1)\end{array}$ & $\begin{array}{l}-0.133055 \\
(0.020931) \\
{[-6.357]^{* * *}}\end{array}$ & $\begin{array}{c}0.0579041 \\
(0.03756) \\
{[1.541]}\end{array}$ & $\begin{array}{c}0.123758 \\
(0.0194174) \\
{[6.374]^{* * *}}\end{array}$ & $\begin{array}{c}-0.176571 \\
(0.0231630) \\
{[-7.623]^{* * *}}\end{array}$ & $\begin{array}{c}0.978478 \\
(0.00574166) \\
{[170.4]^{* * *}}\end{array}$ \\
\hline $\begin{array}{c}\text { Straits Times } \\
\text { EGARCH } \\
(1,1) \\
\end{array}$ & $\begin{array}{c}-0.110242 \\
(0.0194876) \\
{[-5.657]^{* * *}}\end{array}$ & $\begin{array}{c}0.0533691 \\
(0.03698) \\
{[1.443]}\end{array}$ & $\begin{array}{c}0.129310 \\
(0.0231160) \\
{[5.594]^{* * *}} \\
\end{array}$ & $\begin{array}{c}-0.0722343 \\
(0.0117771) \\
{[-6.133]^{* * *}}\end{array}$ & $\begin{array}{c}0.992393 \\
0.00315435) \\
{[314.6]^{* * *}}\end{array}$ \\
\hline $\begin{array}{c}\text { SSMI } \\
\text { EGARCH } \\
(1,1) \\
\end{array}$ & $\begin{array}{c}-0.110147 \\
0.0204024) \\
{[-5.399]^{* * *}}\end{array}$ & $\begin{array}{c}-0.033759 \\
(0.03770) \\
{[-0.8953]}\end{array}$ & $\begin{array}{c}0.151578 \\
(0.0257497) \\
{[5.887]^{* * *}}\end{array}$ & $\begin{array}{c}-0.154043 \\
(0.0202074) \\
{[-7.623]^{* * *}}\end{array}$ & $\begin{array}{c}0.970687 \\
(0.00698062) \\
{[139.1]^{* * *}}\end{array}$ \\
\hline $\begin{array}{c}\text { TAIEX } \\
\text { EGARCH } \\
(1,1) \\
\end{array}$ & $\begin{array}{c}-0.102730 \\
0.0210484) \\
{[-4.881]^{* * *}}\end{array}$ & $\begin{array}{c}0.0248115 \\
(0.03558) \\
{[0.6973}\end{array}$ & $\begin{array}{c}0.136119 \\
(0.0277116) \\
{[4.912]^{* * *}}\end{array}$ & $\begin{array}{c}-0.0740962 \\
(0.0196892) \\
{[-3.763]^{* * *}}\end{array}$ & $\begin{array}{c}0.985772 \\
(0.00679591) \\
{[145.1]^{* * *}}\end{array}$ \\
\hline
\end{tabular}

Notes: Standard errors in round brackets; z-statistics in square brackets; ${ }^{* *},{ }^{* *}, *$ mean significant at $0.01,0.05$, and 0.1 levels, respectively.

Table 8 - Conditional mean equation for the returns of emerging markets indexes from the first sub-sample

\begin{tabular}{|c|c|c|c|}
\hline Index & Constant term & $\begin{array}{c}\text { Coefficient of TOM dummy } \\
\text { variable }\end{array}$ & First order lagged returns \\
\hline Athex Composite & 0.0108693 & 0.103266 & 0.0982412 \\
Share & {$[0.0275089]$} & {$[0.0625302)$} & {$[0.0239255)$} \\
& {$[0.3951]$} & {$[1.651]^{*}$} & {$[4.106]^{* * *}$} \\
\hline BET-C & 0.0926022 & 0.119873 & 0.151954 \\
& {$[0.0250375)$} & {$[0.0596539)$} & {$[0.0265772)$} \\
& {$[3.699]^{* * *}$} & {$[2.009]^{* *}$} & {$[5.717]^{* * *}$} \\
\hline Bovespa & 0.0329743 & 0.386544 & \\
& {$[0.0400274)$} & {$[0.101063)$} & \\
& {$[0.8238]$} & {$[3.825]^{* * *}$} & \\
\hline
\end{tabular}


International Conference "Risk in Contemporary Economy"

ISSN online 2344-5386 ISSN print 2067-0532

XIVth Edition, 2013, Galati, Romania,

"Dunarea de Jos" University of Galati - Faculty of Economics and Business Administration

\begin{tabular}{|c|c|c|c|}
\hline BSE 30 & $\begin{array}{c}0.0263449 \\
(0.0283675) \\
{[0.9287]} \\
\end{array}$ & $\begin{array}{c}0.242269 \\
(0.0690618) \\
{[3.508]^{* * *}} \\
\end{array}$ & $\begin{array}{c}0.100178 \\
(0.0272281) \\
{[3.679]^{* * *}}\end{array}$ \\
\hline BUX & $\begin{array}{c}0.0429649 \\
(0.0326348) \\
{[1.317]}\end{array}$ & $\begin{array}{c}0.138534 \\
(0.0729495) \\
{[1.899]^{*}}\end{array}$ & $\mathrm{x}$ \\
\hline CROBEX & $\begin{array}{c}0.0692799 \\
(0.0266509) \\
{[2.600]^{* * *}} \\
\end{array}$ & $\begin{array}{c}0.0496981 \\
(0.0563450) \\
{[0.8820]} \\
\end{array}$ & $\mathrm{x}$ \\
\hline IDX Composite & $\begin{array}{c}0.0538407 \\
(0.0335790) \\
{[1.603]}\end{array}$ & $\begin{array}{c}0.100256 \\
(0.0513968) \\
{[1.951]^{*}}\end{array}$ & $\begin{array}{c}0.102552 \\
(0.0262306) \\
{[3.910]^{* * *}}\end{array}$ \\
\hline IPC & $\begin{array}{c}0.0593891 \\
(0.0272313) \\
{[2.181]^{* *}} \\
\end{array}$ & $\begin{array}{c}0.199258 \\
(0.0655341) \\
{[3.041]^{* * *}} \\
\end{array}$ & $\begin{array}{c}0.0856522 \\
(0.0235351) \\
{[3.639]^{* * *}}\end{array}$ \\
\hline KLSE Composite & $\begin{array}{c}0.0109393 \\
(0.0149909) \\
{[0.7297]}\end{array}$ & $\begin{array}{c}0.0349013 \\
(0.0368244) \\
{[0.9478]}\end{array}$ & $\begin{array}{c}0.145427 \\
(0.0260489) \\
{[5.583]^{* * *}}\end{array}$ \\
\hline KOSPI & $\begin{array}{c}0.0171869 \\
(0.000041) \\
{[1386]^{* * *}}\end{array}$ & $\begin{array}{c}0.223538 \\
(0.0702143) \\
{[3.184]^{* * *}} \\
\end{array}$ & $\begin{array}{c}0.0144342 \\
(0.000028) \\
{[2274]^{* * *}}\end{array}$ \\
\hline MerVal & $\begin{array}{c}0.0563620 \\
(0.0472894) \\
{[1.192]}\end{array}$ & $\begin{array}{c}0.166789 \\
(0.102677) \\
{[1.624]}\end{array}$ & $\begin{array}{c}0.0409841 \\
(0.0231121) \\
{[1.773]^{*}}\end{array}$ \\
\hline OMXT & $\begin{array}{c}0.0500371 \\
(0.0177996) \\
{[2.811]^{* * *}}\end{array}$ & $\begin{array}{c}0.0172080 \\
(0.0341026) \\
{[0.5046]}\end{array}$ & $\begin{array}{c}0.179138 \\
(0.0286130) \\
{[6.261]^{* * *}}\end{array}$ \\
\hline PX & $\begin{array}{c}0.0850995 \\
(0.0285709) \\
{[2.979]^{* * *}}\end{array}$ & $\begin{array}{c}0.0956345 \\
(0.0673557) \\
{[1.420]}\end{array}$ & $\begin{array}{c}0.0504818 \\
(0.0250193) \\
{[2.018]^{* *}}\end{array}$ \\
\hline SEMDEX & $\begin{array}{c}0.0228857 \\
(0.00892500) \\
{[2.564]^{* *}}\end{array}$ & $\begin{array}{c}0.00659727 \\
(0.0180554) \\
{[0.3654]}\end{array}$ & $\begin{array}{c}0.234309 \\
(0.0226499) \\
{[10.34]^{* * *}}\end{array}$ \\
\hline SSE Composite & $\begin{array}{c}-0.0111683 \\
(0.0329074) \\
{[-0.3394]}\end{array}$ & $\begin{array}{c}0.116634 \\
(0.0481608) \\
{[2.422]^{* *}}\end{array}$ & $\mathrm{x}$ \\
\hline TA 100 & $\begin{array}{c}-0.00149973 \\
(0.000412928) \\
{[-3.632]^{* * *}}\end{array}$ & $\begin{array}{c}0.189240 \\
(0.000016) \\
{[4.841]^{* * *}}\end{array}$ & $\mathrm{x}$ \\
\hline
\end{tabular}

Notes: Standard errors in round brackets; z-statistics in square brackets; ${ }^{* * *},{ }^{* *}, *$ mean significant at $0.01,0.05$, and 0.1 levels, respectively.

Table 9 - Conditional variance equation for the returns of emerging markets indexes from the first subsample

\begin{tabular}{|c|c|c|c|c|c|}
\hline Index & $\begin{array}{l}\text { Constant } \\
\text { term }\end{array}$ & $\begin{array}{c}\text { Coefficient of } \\
\text { TOM dummy } \\
\text { variable }\end{array}$ & alpha & gamma & beta \\
\hline $\begin{array}{c}\text { Athex } \\
\text { Composite } \\
\text { Share } \\
\text { GARCH }(1,1)\end{array}$ & $\begin{array}{c}0.0586448 \\
(0.0428926) \\
{[1.367]}\end{array}$ & $\begin{array}{c}0.0404459 \\
(0.0618711) \\
{[0.6537]}\end{array}$ & $\begin{array}{c}0.112496 \\
(0.0438329) \\
{[2.566]^{* *}}\end{array}$ & $\mathrm{x}$ & $\begin{array}{c}0.850371 \\
(0.067988) \\
{[12.51]^{* * *}}\end{array}$ \\
\hline $\begin{array}{c}\text { BET-C } \\
\text { GARCH }(1,1)\end{array}$ & $\begin{array}{c}0.105236 \\
(0.0472876) \\
{[2.225]^{* *}} \\
\end{array}$ & $\begin{array}{c}0.161592 \\
(0.0743432) \\
{[2.174]^{* *}}\end{array}$ & $\begin{array}{c}0.255013 \\
(0.0600202) \\
{[4.249]^{* * *}}\end{array}$ & $\mathrm{x}$ & $\begin{array}{c}0.687830 \\
(0.0707877) \\
{[9.717]^{* * *}} \\
\end{array}$ \\
\hline $\begin{array}{c}\text { Bovespa } \\
\text { GARCH }(1,1)\end{array}$ & $\begin{array}{c}0.0359241 \\
(0.0316454)\end{array}$ & $\begin{array}{c}0.107526 \\
(0.095746)\end{array}$ & $\begin{array}{c}0.0529704 \\
(0.0119575)\end{array}$ & $\mathrm{x}$ & $\begin{array}{c}0.929732 \\
(0.0169635)\end{array}$ \\
\hline
\end{tabular}


"Dunarea de Jos" University of Galati - Faculty of Economics and Business Administration

\begin{tabular}{|c|c|c|c|c|c|}
\hline & [1.135] & [1.123] & {$[4.430]^{* * *}$} & & {$[54.81]^{* * *}$} \\
\hline $\begin{array}{c}\text { BSE } 30 \\
\text { EGARCH }(1,1)\end{array}$ & $\begin{array}{c}-0.175239 \\
(0.0268250) \\
{[-6.533]^{* * *}}\end{array}$ & $\begin{array}{c}-0.0251110 \\
(0.0305464) \\
{[-0.8221]}\end{array}$ & $\begin{array}{c}0.285238 \\
(0.0425689) \\
{[6.701]^{* * *}}\end{array}$ & $\begin{array}{c}-0.140163 \\
(0.0319006) \\
{[-4.394]^{* * *}}\end{array}$ & $\begin{array}{c}0.913045 \\
(0.0255883) \\
{[35.68]^{* * *}}\end{array}$ \\
\hline $\begin{array}{c}\text { BUX } \\
\text { EGARCH }(1,1)\end{array}$ & $\begin{array}{c}-0.0945660 \\
(0.0221521) \\
{[-4.269]^{* * *}}\end{array}$ & $\begin{array}{c}0.0664718 \\
(0.0392598) \\
{[1.693]^{*}}\end{array}$ & $\begin{array}{c}0.139929 \\
(0.0279247) \\
{[5.011]^{* * *}}\end{array}$ & $\begin{array}{c}-0.0502372 \\
(0.0171774) \\
{[-2.925]^{* * *}}\end{array}$ & $\begin{array}{c}0.955353 \\
(0.0131291) \\
{[72.77]^{* * *}}\end{array}$ \\
\hline $\begin{array}{c}\text { CROBEX } \\
\text { GARCH }(1,1)\end{array}$ & $\begin{array}{c}0.145688 \\
(0.0788154) \\
{[1.848]^{*}}\end{array}$ & $\begin{array}{c}0.0838147 \\
(0.0645421) \\
{[1.299]}\end{array}$ & $\begin{array}{c}0.198607 \\
(0.0661962) \\
{[3.000]^{* * *}}\end{array}$ & $\mathrm{x}$ & $\begin{array}{c}0.726152 \\
(0.0946816) \\
{[7.669]^{* * *}}\end{array}$ \\
\hline $\begin{array}{l}\text { IDX Composite } \\
\text { EGARCH }(1,1)\end{array}$ & $\begin{array}{c}-0.102067 \\
(0.0309236) \\
{[-3.301]^{* * *}}\end{array}$ & $\begin{array}{c}0.100256 \\
(0.0513968) \\
{[1.951]^{*}}\end{array}$ & $\begin{array}{c}0.185209 \\
(0.0452181) \\
{[4.096]^{* * *}}\end{array}$ & $\begin{array}{c}-0.101242 \\
(0.0322398) \\
{[-3.140]^{* * *}}\end{array}$ & $\begin{array}{c}0.885044 \\
(0.0464392) \\
{[19.06]^{* * *}}\end{array}$ \\
\hline $\begin{array}{c}\text { IPC } \\
\text { EGARCH }(1,1)\end{array}$ & $\begin{array}{c}-0.116939 \\
(0.0215523) \\
{[-5.426]^{* * *}}\end{array}$ & $\begin{array}{c}0.0618028 \\
(0.0353247) \\
{[1.750]^{*}}\end{array}$ & $\begin{array}{c}0.149655 \\
(0.0263205) \\
{[5.686]^{* * *}}\end{array}$ & $\begin{array}{c}-0.104035 \\
(0.0186929) \\
{[-5.565]^{* * *}}\end{array}$ & $\begin{array}{c}0.970080 \\
(0.00853130) \\
{[113.7]^{* * *}}\end{array}$ \\
\hline $\begin{array}{c}\text { KLSE } \\
\text { Composite } \\
\text { EGARCH }(1,1)\end{array}$ & $\begin{array}{c}-0.197514 \\
(0.0522791) \\
{[-3.778]^{* * *}}\end{array}$ & $\begin{array}{c}0.0762703 \\
(0.0423468) \\
{[1.801]^{*}}\end{array}$ & $\begin{array}{c}0.224710 \\
(0.0596599) \\
{[3.767]^{* * *}}\end{array}$ & $\begin{array}{c}-0.0431858 \\
(0.0185875) \\
{[-2.323]^{* *}}\end{array}$ & $\begin{array}{c}0.972862 \\
(0.0142098) \\
{[68.46]^{* * *}}\end{array}$ \\
\hline $\begin{array}{c}\text { KOSPI } \\
\text { EGARCH }(1,1)\end{array}$ & $\begin{array}{c}-0.0928303 \\
(0.0193030) \\
{[-4.809]^{* * *}}\end{array}$ & $\begin{array}{c}-0.00823267 \\
0.0433771) \\
{[-0.1898]}\end{array}$ & $\begin{array}{c}0.145809 \\
(0.0306315) \\
{[4.760]^{* * *}}\end{array}$ & $\begin{array}{c}-0.0768932 \\
(0.0219416) \\
{[-3.504]^{* * *}}\end{array}$ & $\begin{array}{c}0.981185 \\
(0.00800805) \\
{[122.5]^{* * *}}\end{array}$ \\
\hline $\begin{array}{c}\text { MerVal } \\
\text { GJR GARCH } \\
(1,1)\end{array}$ & $\begin{array}{c}0.0922922 \\
(0.0380726) \\
{[2.424]^{* *}}\end{array}$ & $\begin{array}{c}0.102929 \\
(0.111867) \\
{[0.9201]}\end{array}$ & $\begin{array}{c}0.0898320 \\
(0.0154424) \\
{[5.817]^{* * *}}\end{array}$ & $\begin{array}{c}0.243803 \\
(0.0684059) \\
{[3.564]^{* * *}}\end{array}$ & $\begin{array}{c}0.882846 \\
(0.0187946) \\
{[46.97]^{* * *}}\end{array}$ \\
\hline $\begin{array}{c}\text { OMXT } \\
\text { GARCH }(1,1)\end{array}$ & $\begin{array}{c}0.0176086 \\
(0.00780573) \\
{[2.256]^{* *}}\end{array}$ & $\begin{array}{c}-0.0261127 \\
(0.0152966) \\
{[-1.707]^{*}}\end{array}$ & $\begin{array}{c}0.195381 \\
(0.0398086) \\
{[4.908]^{* * *}}\end{array}$ & $\mathrm{x}$ & $\begin{array}{c}0.831060 \\
(0.0281272) \\
{[29.55]^{* * *}}\end{array}$ \\
\hline $\begin{array}{c}\text { PX } \\
\text { GJR GARCH } \\
(1,1)\end{array}$ & $\begin{array}{c}0.0597500 \\
(0.0219326) \\
{[2.724]^{* * *}}\end{array}$ & $\begin{array}{c}-0.00357790 \\
(0.0490258) \\
{[-0.07298]}\end{array}$ & $\begin{array}{c}0.0918586 \\
(0.0142770) \\
{[6.434]^{* * *}}\end{array}$ & $\begin{array}{c}0.286176 \\
(0.0945298) \\
{[3.027]^{* * *}}\end{array}$ & $\begin{array}{c}0.860557 \\
(0.0200696) \\
{[42.88]^{* * *}}\end{array}$ \\
\hline $\begin{array}{c}\text { SEMDEX } \\
\text { EGARCH }(1,1)\end{array}$ & $\begin{array}{c}-0.539155 \\
(0.0700498) \\
{[-7.697]^{* * *}}\end{array}$ & $\begin{array}{c}0.139977 \\
(0.0572962) \\
{[2.443]^{* *}}\end{array}$ & $\begin{array}{c}0.419315 \\
(0.0564841) \\
{[7.424]^{* * *}}\end{array}$ & $\begin{array}{c}0.0899361 \\
(0.0315990) \\
{[2.846]^{* * *}}\end{array}$ & $\begin{array}{c}0.860871 \\
(0.0270596) \\
{[31.81]^{* * *}}\end{array}$ \\
\hline $\begin{array}{c}\text { SSE Composite } \\
\text { GARCH }(1,1)\end{array}$ & $\begin{array}{c}0.298314 \\
(0.119167) \\
{[2.503]^{* *}}\end{array}$ & $\begin{array}{c}-0.269371 \\
(0.154192) \\
{[-1.747]^{*}}\end{array}$ & $\begin{array}{c}0.207648 \\
(0.0680928) \\
{[3.049]^{* * *}}\end{array}$ & $\mathrm{x}$ & $\begin{array}{c}0.725401 \\
(0.0661642) \\
{[10.96]^{* * *}}\end{array}$ \\
\hline $\begin{array}{c}\text { TA } 100 \\
\text { EGARCH }(1,1)\end{array}$ & $\begin{array}{c}-0.121981 \\
(0.0271157) \\
{[-4.499]^{* * *}}\end{array}$ & $\begin{array}{c}0.0638609 \\
(0.0531025) \\
{[1.203]}\end{array}$ & $\begin{array}{c}0.178918 \\
(0.0368355) \\
{[4.857]^{* * *}}\end{array}$ & $\begin{array}{c}-0.0719682 \\
(0.0262554) \\
{[-2.741]^{* * *}}\end{array}$ & $\begin{array}{c}0.948836 \\
(0.0193486) \\
{[49.04]^{* * *}}\end{array}$ \\
\hline
\end{tabular}

Notes: Standard errors in round brackets; z-statistics in square brackets; ${ }^{* * *},{ }^{* *},{ }^{*}$ mean significant at 0.01 , 0.05 , and 0.1 levels, respectively.

Table 10 - Conditional mean equation for the returns of emerging markets indexes from the second subsample

\begin{tabular}{|c|c|c|c|}
\hline Index & Constant term & $\begin{array}{c}\text { Coefficient of TOM dummy } \\
\text { variable }\end{array}$ & $\begin{array}{c}\text { First order lagged } \\
\text { returns }\end{array}$ \\
\hline Athex Composite & -0.0564631 & 0.194571 & 0.0538544 \\
Share & {$[0.0463139)$} & {$[0.101326)$} & {$[2.0227961)$} \\
& {$[-1.219]$} & {$[1.920]^{*}$} & 0.0792718 \\
\hline BET-C & -0.00861223 & 0.162227 & {$[0.0261177)$} \\
& {$[0.025648)$} & {$[0.0647087)$} & {$[3.035]^{* * *}$} \\
\hline Bovespa & {$[-0.3358]$} & {$[2.507]^{* *}$} & $\mathrm{x}$ \\
\hline
\end{tabular}


"Dunarea de Jos" University of Galati - Faculty of Economics and Business Administration

\begin{tabular}{|c|c|c|c|}
\hline BSE 30 & $\begin{array}{c}-0.0243808 \\
(0.0325835) \\
{[-0.7483]}\end{array}$ & $\begin{array}{c}0.165797 \\
(0.0660482) \\
{[2.510]^{* *}}\end{array}$ & $\begin{array}{c}0.0525280 \\
(0.0221255) \\
{[2.374]^{* *}}\end{array}$ \\
\hline BUX & $\begin{array}{c}-0.0484894 \\
(0.0336533) \\
{[-1.441]}\end{array}$ & $\begin{array}{c}0.154997 \\
(0.0730407) \\
{[2.122]^{* *}}\end{array}$ & $\mathrm{x}$ \\
\hline CROBEX & $\begin{array}{c}-0.0435305 \\
(0.0213535) \\
{[-2.039]^{* *}}\end{array}$ & $\begin{array}{c}0.111569 \\
{[0.0479347} \\
{[2.328]^{* *}} \\
\end{array}$ & $\begin{array}{c}0.0976693 \\
(0.0247232) \\
{[3.951]^{* * *}} \\
\end{array}$ \\
\hline IDX Composite & $\begin{array}{c}0.0414456 \\
(0.0295099) \\
{[1.404]}\end{array}$ & $\begin{array}{c}0.111204 \\
(0.0670483) \\
{[1.659]^{*}} \\
\end{array}$ & $\mathrm{x}$ \\
\hline IPC & $\begin{array}{c}0.000477262 \\
(0.0263725) \\
{[0.01810]}\end{array}$ & $\begin{array}{c}0.183240 \\
(0.0599256) \\
{[3.058]^{* * *}}\end{array}$ & $\mathrm{x}$ \\
\hline KLSE Composite & $\begin{array}{c}0.0176274 \\
(0.0162320) \\
{[1.086]} \\
\end{array}$ & $\begin{array}{c}0.0797189 \\
(0.0420994) \\
{[1.894]^{*}}\end{array}$ & $\begin{array}{c}0.103882 \\
(0.0273801) \\
{[3.794]^{* * *}} \\
\end{array}$ \\
\hline KOSPI & $\begin{array}{c}0.000479033 \\
(0.0281488) \\
{[0.01702]}\end{array}$ & $\begin{array}{c}0.112812 \\
(0.0637529) \\
{[1.770]^{*}}\end{array}$ & $\mathrm{x}$ \\
\hline MerVal & $\begin{array}{c}0.0478333 \\
(0.0475773) \\
{[1.005]}\end{array}$ & $\begin{array}{c}0.106920 \\
(0.0926417) \\
{[1.154]}\end{array}$ & $\mathrm{x}$ \\
\hline OMXT & $\begin{array}{c}0.00356583 \\
(0.0240796) \\
{[0.1481]}\end{array}$ & $\begin{array}{c}0.0836532 \\
(0.0479991) \\
{[1.743]^{*}}\end{array}$ & $\mathrm{x}$ \\
\hline PX & $\begin{array}{c}-0.0358851 \\
(0.0303009) \\
{[-1.184]}\end{array}$ & $\begin{array}{c}0.174214 \\
(0.0632501) \\
{[2.754]^{* * *}} \\
\end{array}$ & $\mathrm{x}$ \\
\hline SEMDEX & $\begin{array}{c}0.0181952 \\
(0.0118623) \\
{[1.534]}\end{array}$ & $\begin{array}{c}0.0329361 \\
(0.0229163) \\
{[1.437]}\end{array}$ & $\begin{array}{c}0.309609 \\
(0.0271780) \\
{[11.39]^{* * *}} \\
\end{array}$ \\
\hline SSE Composite & $\begin{array}{c}-0.0655578 \\
(0.0426259) \\
{[-1.538]}\end{array}$ & $\begin{array}{c}0.181188 \\
(0.112472) \\
{[1.611]}\end{array}$ & $\mathrm{x}$ \\
\hline TA 100 & $\begin{array}{c}0.00848683 \\
(0.0287772) \\
{[0.2949]}\end{array}$ & $\begin{array}{c}0.106732 \\
(0.0617736) \\
{[1.728]^{*}}\end{array}$ & $\begin{array}{c}-0.136644 \\
{[0.0259431} \\
{[-5.267]^{* * *}}\end{array}$ \\
\hline
\end{tabular}

Notes: Standard errors in round brackets; z-statistics in square brackets; ${ }^{* * *}, * *, *$ mean significant at 0.01 , 0.05 , and 0.1 levels, respectively.

Table 11 - Conditional variance equation for the returns of emerging markets indexes from the second sub-sample

\begin{tabular}{|c|c|c|c|c|c|}
\hline Index & Constant term & $\begin{array}{c}\text { Coefficient of } \\
\text { TOM dummy } \\
\text { variable }\end{array}$ & alpha & gamma & beta \\
\hline Athex Composite & -0.128186 & 0.0537454 & 0.206473 & -0.0489078 & 0.972073 \\
Share & {$[0.0219227)$} & {$[0.0377380)$} & {$[0.0305723)$} & {$[0.0173253)$} & $(0.0081858)$ \\
EGARCH (1,1) & {$[-5.847]^{* * *}$} & {$[1.424]$} & {$[6.754]^{* * *}$} & {$[-2.823]^{* * *}$} & {$[118.8]^{* * *}$} \\
\hline BET-C & 0.0204426 & 0.0621684 & 0.151441 & $\mathrm{x}$ & 0.844614 \\
GARCH (1,1) & {$[0.0149498)$} & {$[0.0393804)$} & {$[0.0367025)$} & & {$[0.0359508)$} \\
& {$[1.367]$} & {$[1.579]$} & {$[4.126]^{* * *}$} & & {$[23.49]^{* * *}$} \\
\hline Bovespa & 0.0407206 & 0.0758472 & 0.0565567 & 0.137681 & 0.905886 \\
GJR GARCH (1,1) & {$[0.0311212)$} & {$[0.0701436)$} & {$[0.0201379)$} & {$[0.039990)$} & $(0.0293826)$ \\
& {$[1.308]$} & {$[1.081]$} & {$[2.808]^{* * *}$} & {$[3.443]^{* * *}$} & {$[30.83]^{* * *}$} \\
\hline
\end{tabular}


"Dunarea de Jos" University of Galati - Faculty of Economics and Business Administration

\begin{tabular}{|c|c|c|c|c|c|}
\hline $\begin{array}{c}\text { BSE } 30 \\
\text { EGARCH }(1,1)\end{array}$ & $\begin{array}{c}-0.113958 \\
(0.0211986) \\
{[-5.376]^{* * *}}\end{array}$ & $\begin{array}{c}-0.0329128 \\
(0.0451324) \\
{[-0.7293]}\end{array}$ & $\begin{array}{c}0.172789 \\
(0.0286612) \\
{[6.029]^{* * *}}\end{array}$ & $\begin{array}{c}-0.102715 \\
(0.0204374) \\
{[-5.026]^{* * *}}\end{array}$ & $\begin{array}{c}0.983505 \\
(0.0059445) \\
{[165.4]^{* * *}}\end{array}$ \\
\hline $\begin{array}{c}\text { BUX } \\
\text { EGARCH }(1,1)\end{array}$ & $\begin{array}{c}-0.125241 \\
(0.0218901) \\
{[-5.721]^{* * *}}\end{array}$ & $\begin{array}{c}-0.00827314 \\
(0.0411378) \\
{[-0.2011]}\end{array}$ & $\begin{array}{c}0.181461 \\
(0.0295289) \\
{[6.145]^{* * *}}\end{array}$ & $\begin{array}{c}-0.0598784 \\
(0.0151099) \\
{[-3.963]^{* * *}}\end{array}$ & $\begin{array}{c}0.983873 \\
(0.0065571) \\
{[150.0]^{* * *}}\end{array}$ \\
\hline $\begin{array}{c}\text { CROBEX } \\
\text { GJR GARCH }(1,1)\end{array}$ & $\begin{array}{c}0.00594874 \\
(0.00685366) \\
{[0.8680]}\end{array}$ & $\begin{array}{c}0.0108875 \\
(0.0269027) \\
{[0.4047]}\end{array}$ & $\begin{array}{c}0.0737943 \\
(0.0220162) \\
{[3.352]^{* * *}}\end{array}$ & $\begin{array}{c}0.211292 \\
(0.0695012) \\
{[3.040]^{* * *}}\end{array}$ & $\begin{array}{c}0.920461 \\
(0.0204963) \\
{[44.91]^{* * *}}\end{array}$ \\
\hline $\begin{array}{c}\text { IDX Composite } \\
\text { GJR GARCH }(1,1)\end{array}$ & $\begin{array}{c}0.0713572 \\
(0.0390590) \\
{[1.827]^{*}}\end{array}$ & $\begin{array}{c}0.0626008 \\
(0.0590628) \\
{[1.060]}\end{array}$ & $\begin{array}{c}0.132421 \\
(0.0357216) \\
{[3.707]^{* * *}}\end{array}$ & $\begin{array}{c}0.312538 \\
(0.0766005) \\
{[4.080]^{* * *}}\end{array}$ & $\begin{array}{c}0.820454 \\
(0.0524580) \\
{[15.64]^{* * *}}\end{array}$ \\
\hline $\begin{array}{c}\text { IPC } \\
\text { GARCH }(1,1)\end{array}$ & $\begin{array}{c}0.0211372 \\
(0.0103768) \\
{[2.037]^{* *}} \\
\end{array}$ & $\begin{array}{c}-0.0312469 \\
(0.0425291) \\
{[-0.7347]}\end{array}$ & $\begin{array}{c}0.0871723 \\
(0.0159793) \\
{[5.455]^{* * *}}\end{array}$ & $\mathrm{x}$ & $\begin{array}{c}0.906602 \\
(0.0158493) \\
{[57.20]^{* * *}}\end{array}$ \\
\hline $\begin{array}{l}\text { KLSE Composite } \\
\text { GJR GARCH }(1,1)\end{array}$ & $\begin{array}{c}0.0533215 \\
(0.0222640) \\
{[2.395]^{* *}} \\
\end{array}$ & $\begin{array}{c}0.0284713 \\
(0.0286321) \\
{[0.9944]}\end{array}$ & $\begin{array}{c}0.261608 \\
(0.0564473) \\
{[4.635]^{* * *}}\end{array}$ & $\begin{array}{c}0.209763 \\
(0.0590137) \\
{[3.554]^{* * *}} \\
\end{array}$ & $\begin{array}{c}0.696763 \\
(0.0617251) \\
{[11.29]^{* * *}}\end{array}$ \\
\hline $\begin{array}{c}\text { KOSPI } \\
\text { GJR GARCH }(1,1)\end{array}$ & $\begin{array}{c}0.0443324 \\
(0.0177432) \\
{[2.499]^{* *}}\end{array}$ & $\begin{array}{c}-0.00554802 \\
(0.0558465) \\
{[-0.09934]}\end{array}$ & $\begin{array}{c}0.0421867 \\
(0.0082559) \\
{[5.110]^{* * *}}\end{array}$ & $\begin{array}{c}1.01996 \\
(0.0444612) \\
{[22.94]^{* * *}}\end{array}$ & $\begin{array}{c}0.887730 \\
(0.0193146) \\
{[45.96]^{* * *}}\end{array}$ \\
\hline $\begin{array}{c}\text { MerVal } \\
\text { GARCH }(1,1)\end{array}$ & $\begin{array}{c}0.0751617 \\
(0.0566648) \\
{[1.326]}\end{array}$ & $\begin{array}{c}0.199390 \\
(0.139877) \\
{[1.425]}\end{array}$ & $\begin{array}{c}0.0965518 \\
(0.0257012) \\
{[3.757]^{* * *}}\end{array}$ & $\mathrm{x}$ & $\begin{array}{c}0.877533 \\
(0.0337679) \\
{[25.99]^{* * *}}\end{array}$ \\
\hline $\begin{array}{c}\text { OMXT } \\
\text { GARCH }(1,1)\end{array}$ & $\begin{array}{c}0.0170777 \\
(0.0141632) \\
{[1.206]}\end{array}$ & $\begin{array}{c}0.0637431 \\
(0.0439766) \\
{[1.449]}\end{array}$ & $\begin{array}{c}0.158354 \\
(0.0462826) \\
{[3.421]^{* * *}}\end{array}$ & $\mathrm{x}$ & $\begin{array}{c}0.844664 \\
(0.0412746) \\
{[20.46]^{* * *}}\end{array}$ \\
\hline $\begin{array}{c}\text { PX } \\
\text { EGARCH }(1,1)\end{array}$ & $\begin{array}{c}-0.179092 \\
(0.0252977) \\
{[-7.079]^{* * *}}\end{array}$ & $\begin{array}{c}0.00547726 \\
(0.0392186) \\
{[0.1397]}\end{array}$ & $\begin{array}{c}0.245214 \\
(0.0329646) \\
{[7.439]^{* * *}}\end{array}$ & $\begin{array}{c}-0.0556826 \\
(0.0174221) \\
{[-3.196]^{* * *}}\end{array}$ & $\begin{array}{c}0.975567 \\
(0.0073921) \\
{[132.0]^{* * *}}\end{array}$ \\
\hline $\begin{array}{c}\text { SEMDEX } \\
\text { GARCH }(1,1)\end{array}$ & $\begin{array}{c}0.0236321 \\
(0.00979674) \\
{[2.412]^{* *}}\end{array}$ & $\begin{array}{c}-0.00637919 \\
(0.0105269) \\
{[-0.6060]}\end{array}$ & $\begin{array}{c}0.331147 \\
(0.0932574) \\
{[3.551]^{* * *}}\end{array}$ & $\mathrm{x}$ & $\begin{array}{c}0.691324 \\
(0.0747071) \\
{[9.254]^{* * *}}\end{array}$ \\
\hline $\begin{array}{c}\text { SSE Composite } \\
\text { GARCH }(1,1)\end{array}$ & $\begin{array}{c}0.00316801 \\
(0.0107690) \\
{[0.2942]}\end{array}$ & $\begin{array}{c}0.00810933 \\
(0.0460545) \\
{[0.1761]}\end{array}$ & $\begin{array}{c}0.0268869 \\
(0.0093494) \\
{[2.876]^{* * *}}\end{array}$ & $\mathrm{x}$ & $\begin{array}{c}0.971985 \\
(0.0098928) \\
{[98.25]^{* * *}}\end{array}$ \\
\hline $\begin{array}{c}\text { TA } 100 \\
\text { EGARCH }(1,1)\end{array}$ & $\begin{array}{c}-0.0975141 \\
(0.0291778) \\
{[-3.342]^{* * *}}\end{array}$ & $\begin{array}{c}-0.0182498 \\
(0.0598561) \\
{[-0.3049]}\end{array}$ & $\begin{array}{c}0.140574 \\
(0.0402330) \\
{[3.494]^{* * *}}\end{array}$ & $\begin{array}{c}-0.0704078 \\
(0.016673) \\
{[-4.223]^{* * *}}\end{array}$ & $\begin{array}{c}0.990158 \\
(0.0059144) \\
{[167.4]^{* * *}}\end{array}$ \\
\hline
\end{tabular}

Notes: Standard errors in round brackets; z-statistics in square brackets; ${ }^{* * *},{ }^{* *}, *$ mean significant at 0.01 , 0.05 , and 0.1 levels, respectively.

Table 12 - Results of ARCH LM tests for residuals of GARCH models

\begin{tabular}{|c|c|c|c|c|c|}
\hline \multicolumn{3}{|c|}{ Advanced Markets } & \multicolumn{3}{c|}{ Emerging Markets } \\
\hline Index & $\begin{array}{c}\text { First sub- } \\
\text { sample }\end{array}$ & $\begin{array}{c}\text { Second sub- } \\
\text { sample }\end{array}$ & Index & $\begin{array}{c}\text { First sub- } \\
\text { sample }\end{array}$ & $\begin{array}{c}\text { Second sub- } \\
\text { sample }\end{array}$ \\
\hline AEX General & 3.8243 & 8.6242 & Athex Composite & 4.716 & 8.412 \\
& $\{0.5731\}$ & $\{0.2146\}$ & Share & $\{0.7308\}$ & $\{0.7385\}$ \\
\hline All Ordinaries & 5.1643 & 6.8621 & BET-C & 6.493 & 6.247 \\
& $\{0.8604\}$ & $\{0.5869\}$ & & $\{0.6975\}$ & $\{0.5942\}$ \\
\hline ATX & 5.8408 & 12.1661 & Bovespa & 8.884 & 8.741 \\
& $\{0.8408\}$ & $\{0.3141\}$ & & $\{0.6851\}$ & $\{0.6475\}$ \\
\hline BEL-20 & 4.701 & 2.2061 & BSE 30 & 3.496 & 6.478 \\
& $\{0.7374\}$ & $\{0.8741\}$ & & $\{0.7612\}$ & $\{0.6861\}$ \\
\hline CAC 40 & 4.717 & 11.2851 & BUX & 10.638 & 4.825 \\
& $\{0.8361\}$ & $\{0.5011\}$ & & $\{0.5887\}$ & $\{0.758\}$ \\
\hline
\end{tabular}


International Conference "Risk in Contemporary Economy"

ISSN online 2344-5386 ISSN print 2067-0532

XIVth Edition, 2013, Galati, Romania,

"Dunarea de Jos" University of Galati - Faculty of Economics and Business Administration

\begin{tabular}{|c|c|c|c|c|c|}
\hline \multicolumn{7}{|c|}{ DAX } & 5.062 & 7.1241 & CROBEX & 3.384 & 8.614 \\
& $\{0.4906\}$ & $\{0.2873\}$ & & $\{0.7951\}$ & $\{0.6802\}$ \\
\hline FTSE 100 & 5.114 & 7.4616 & IDX Composite & 6.802 & 6.705 \\
& $\{0.6628\}$ & $\{0.5913\}$ & & $\{0.7155\}$ & $\{0.6940\}$ \\
\hline Hang Seng & 3.735 & 11.985 & IPC & 4.275 & 3.710 \\
& $\{0.6874\}$ & $\{0.4907\}$ & & $\{0.7403\}$ & $\{0.7112\}$ \\
\hline FTSE MIB & 3.208 & 6.8251 & KLSE Composite & 10.769 & 7.715 \\
& $\{0.7341\}$ & $\{0.3311\}$ & & $\{0.5751\}$ & $\{0.7301\}$ \\
\hline Nikkei 225 & 5.347 & 8.804 & KOSPI & 4.841 & 10.517 \\
& $\{0.8258\}$ & $\{0.383\}$ & & $\{0.7116\}$ & $\{0.2294\}$ \\
\hline OSEAX & 3.571 & 6.482 & MerVal & 3.516 & 7.161 \\
& $\{0.5970\}$ & $\{0.4216\}$ & & $\{0.6841\}$ & $\{0.2115\}$ \\
\hline S\&P TSX & 2.884 & 8.407 & OMXT & 4.340 & 8.7514 \\
Composite & $\{0.7168\}$ & $\{0.3012\}$ & & $\{0.6853\}$ & $\{0.3501\}$ \\
\hline Standard \& & 5.773 & 6.291 & PX & 10.164 & 8.826 \\
Poor's & $\{0.6249\}$ & $\{0.2946\}$ & & $\{0.5738\}$ & $\{0.6419\}$ \\
\hline Straits Times & 4.259 & 13.196 & SEMDEX & 8.714 & 8.8731 \\
& $\{0.7124\}$ & $\{0.5197\}$ & & $\{0.6428\}$ & $\{0.7943\}$ \\
\hline SSMI & 4.614 & 8.867 & SSE Composite & 6.217 & 7.175 \\
& $\{0.6105\}$ & $\{0.5160\}$ & & $\{0.7510\}$ & $\{0.2961\}$ \\
\hline TAIEX & 4.287 & 11.503 & TA 100 & 3.841 & 6.617 \\
& $\{0.8285\}$ & $\{0.3821\}$ & & $\{0.7322\}$ & $\{0.4612\}$ \\
\hline
\end{tabular}

Note: p-values are within accolads. 